\title{
Robust Smoothed Analysis of a Condition Number for Linear Programming
}

\author{
Peter Bürgisser* and Dennis Amelunxen* \\ University of Paderborn \\ \{pbuerg,damelunx\}@math.upb.de
}

November 3, 2018

\begin{abstract}
We perform a smoothed analysis of the GCC-condition number $\mathscr{C}(A)$ of the linear programming feasibility problem $\exists x \in \mathbb{R}^{m+1} A x<0$. Suppose that $\bar{A}$ is any matrix with rows $\bar{a}_{i}$ of euclidean norm 1 and, independently for all $i$, let $a_{i}$ be a random perturbation of $\bar{a}_{i}$ following the uniform distribution in the spherical disk in $S^{m}$ of angular radius $\arcsin \sigma$ and centered at $\bar{a}_{i}$. We prove that $\mathbb{E}(\ln \mathscr{C}(A))=$ $\mathcal{O}(m n / \sigma)$. A similar result was shown for Renegar's condition number and Gaussian perturbations by Dunagan, Spielman, and Teng [arXiv:cs.DS/0302011]. Our result is robust in the sense that it easily extends to radially symmetric probability distributions supported on a spherical disk of radius $\arcsin \sigma$, whose density may even have a singularity at the center of the perturbation. Our proofs combine ideas from a recent paper of Bürgisser, Cucker, and Lotz (Math. Comp. 77, No. 263, 2008) with techniques of Dunagan et al.
\end{abstract}

AMS subject classifications: 90C05, 90C31, 52A22, 60D05

Key words: linear programming, perturbation, condition number, smoothed analysis, spherically convex sets

\section{Introduction}

A distinctive feature of the computations considered in numerical analysis is that they are affected by errors. A main character in the understanding of

*Institute of Mathematics, University of Paderborn, Germany. Partially supported by DFG grant BU 1371/2-1 and DFG Research Training Group on Scientific Computation GRK 693 (PaSCo GK). 
the effects of these errors is the condition number of the input at hand. This is a positive number measuring the sensitivity of the output with respect to small perturbations of the input. The best known condition number is that for matrix inversion and linear equation solving, which takes the form $\kappa(A)=\|A\|\left\|A^{-1}\right\|$ for a square matrix $A$. Condition numbers not only occur in round-off analysis, but also appear as a parameter in complexity bounds for a variety of iterative algorithms in linear algebra, linear and convex optimization, and polynomial equation solving. Yet, condition numbers are not easily computable. As a way out for this situation, Smale suggested to assume a probability measure on the set of data and to study the condition number of this data as a random variable. Examples of such results abound for a variety of condition numbers. For more details and references we refer to Smale's survey [25] and the recent survey [4].

Renegar $[20,21,22]$ was the first to realize that the computational complexity of linear programming problems can be bounded by a polynomial in the number of variables and inequalities and a certain condition measure of the input. This condition measure is given by the inverse distance of the input to the set of ill-posed systems. More specifically, it is well known that for a given matrix $A \in \mathbb{R}^{n \times(m+1)}$, either the system $A x<0$ or its dual system $A^{T} y=0, y>0$ have a solution, unless we are in an ill-posed situation. The (homogeneous) linear programming feasibility problem is to decide this alternative for given $A$ and to compute a solution of the corresponding system. A primal-dual interior point method is used in [12] to solve the linear programming feasibility problem within

$$
\mathcal{O}(\sqrt{m+n}(\ln (m+n)+\ln \mathscr{C}(A)))
$$

iterations, with each step costing at most $\mathcal{O}\left((m+n)^{3}\right)$ arithmetic operations. Here, the $G C C$-condition number $\mathscr{C}(A)$ is a variant of Renegar's condition number introduced by Goffin [17], and later generalized by Cucker and Cheung [8] (see $\S 2.3$ for the definition). The advantage of $\mathscr{C}(A)$ over Renegar's condition number is that this quantity can be neatly characterized in terms of spherical geometry, which greatly facilitates its probabilistic analysis.

Thus the running time of the primal-dual interior point method used in [12] is controlled by $\mathscr{C}(A)$. Understanding the average-case behaviour of this algorithm therefore boils down to studying $\ln \mathscr{C}(A)$ for random inputs $A$. Motivated by this observation, a lot of efforts have been devoted to the average-case analysis of the random variable $\mathscr{C}(A)$, i.e., to compute the expected value (or the distribution function) of $\ln \mathscr{C}(A)$ for random matrices $A$. In most cases, the matrices $A$ are assumed to have random entries 
which are i.i.d. standard normal. We remark that since the condition number $\mathscr{C}(A)$ is multi-homogeneous in the rows $a_{i}$ of $A$, this is equivalent to considering $\mathscr{C}(A)$ in the case where $a_{1}, \ldots, a_{n}$ are i.i.d. uniformly distributed in unit sphere $S^{m}:=\left\{x \in \mathbb{R}^{m+1} \mid\|x\|=1\right\}$.

The papers dealing with the average analysis of $\mathscr{C}(A)$ are easily summarized. A bound for $\mathbb{E}(\ln \mathscr{C}(A))$ of the form $\mathcal{O}(\min \{n, m \ln n\})$ was shown in [9]. This bound was improved in [13] to $\max \{\ln m, \ln \ln n\}+\mathcal{O}(1)$ assuming that $n$ is moderately larger than $m$. Still, in [10], the asymptotic behavior of both $\mathscr{C}(A)$ and $\ln \mathscr{C}(A)$ was exhaustively studied and these results were extended in [18] to matrices $A \in\left(S^{m}\right)^{n}$ drawn from distributions more general than the uniform. Finally, in [5], the exact distribution of $\mathscr{C}(A)$ conditioned to $A$ being feasible was found and asymptotically sharp tail bounds for the infeasible case were given. In particular, it was shown that $\mathbb{E}(\ln \mathscr{C}(A))=\mathcal{O}(\ln m)$. Our method yields another proof of this result (Cor. 1.3).

\subsection{Smoothed analysis}

The problem of average-case analysis is that its results strongly depend on the distribution of the inputs, which is unknown, and usually assumed to be Gaussian for rendering the mathematical analysis feasible. Spielman and Teng [26, 27, 28] suggested in 2001 the concept of smoothed analysis of algorithms, which is a new form of analysis of algorithms that arguably blends the best of both worst-case and average-case. They used this new framework to give a more compelling explanation of the speed of the simplex method (for the shadow-vertex pivot rule).

The general idea of smoothed analysis is easy to explain. Let $T: \mathbb{R}^{p} \rightarrow$ $\mathbb{R}_{+} \cup\{\infty\}$ be any function (measuring running time, log of condition numbers etc.). Instead of showing "it is unlikely that $T(a)$ will be large," one shows that "for all $\bar{a} \in \mathbb{R}^{p}$ and all slight random perturbations $a$ of $\bar{a}$, it is unlikely that $T(a)$ will be large." If we assume that $a$ is multivariate normal with mean $\bar{a}$ and variance $\sigma^{2}$, in short $a \in N\left(\bar{a}, \sigma^{2}\right)$, then the goal of a smoothed analysis of $T$ is to give good estimates of

$$
\sup _{\bar{a} \in \mathbb{R}^{p}} \operatorname{Prob}_{a \in N\left(\bar{a}, \sigma^{2}\right)}\left\{T(a)>\varepsilon^{-1}\right\} .
$$

In a first approach one may focus on bounds on the expectations.

For many situations of interest, it turns out that in smoothed analysis, there is only a weak dependence on the chosen model of random perturbations. A first formal evidence of this robustness phenomenon was given by 
Cucker, Hauser, and Lotz [11] for certain conic condition numbers, stated below as Theorem 1.1.

Dunagan et al. [15] (see also Spielman and Teng [27]) performed a smoothed analysis of the running time of interior point methods of linear programming by analyzing Renegar's condition number $\mathscr{C}^{\prime}$, a variant of the GCC condition number $\mathscr{C}$. Among other things, they proved the following:

$$
\sup _{\bar{A}} \mathbb{E}_{A \in N\left(\bar{A}, \sigma^{2}\right)}\left(\ln \mathscr{C}^{\prime}(A)\right)=\mathcal{O}\left(\ln \frac{m n}{\sigma}\right) .
$$

Here the supremum is over all $\bar{A} \in \mathbb{R}^{n \times(m+1)}$ of Frobenius norm at most one. Our main result (Theorem 1.2) shows that a similar bound actually holds for a large class of random perturbation laws.

\subsection{A geometric approach to conic condition numbers}

Bürgisser et al. [6, 7] recently extended a result of Demmel [14] on conic condition numbers from average-case analysis to smoothed analysis. There, the perturbations of the inputs are modelled by uniform instead of Gaussian distributions. This allows to perform the analysis in a general geometric framework that we explain next.

The set of ill-posed inputs to a computational problem is modelled as a lower dimensional subset $\Sigma$ of the data space $\mathscr{D}$, which is assumed to be furnished with a metric $d$, a distance function dist (not necessarily a metric), and a volume measure. In our cases of interest, $\mathscr{D}$ is a Riemannian manifold, $d$ is the corresponding metric, and dist $=\sin (d)$. The corresponding condition number $\mathscr{C}(a)$ of an input $a \in \mathscr{D}$ is then defined as

$$
\mathscr{C}(a)=\frac{1}{\operatorname{dist}(a, \Sigma)}
$$

This is an appropriate definition for many applications. In this model, the set of inputs $a$ with condition $\mathscr{C}(a)>\varepsilon^{-1}$ is given by the $\varepsilon$-neighborhood

$$
T_{\text {dist }}(\Sigma, \varepsilon)=\{a \in \mathscr{D} \mid \operatorname{dist}(a, \Sigma)<\varepsilon\} .
$$

Let $B(\bar{a}, \alpha):=\{a \in \mathscr{D} \mid d(a, \bar{a}) \leq \alpha\}$ denote the ball centered at $\bar{a} \in \mathscr{D}$ of radius $\alpha$. The task of a uniform smoothed analysis of $\mathscr{C}$ consists of providing good upper bounds on

$$
\sup _{\bar{a} \in \mathscr{D}} \operatorname{Prob}_{a \in B(\bar{a}, \alpha)}\left\{\mathscr{C}(a)>\varepsilon^{-1}\right\}
$$


where $a$ is assumed to be chosen uniformly at random in $B(\bar{a}, \alpha)$. The probability occurring here thus has an immediate geometric meaning:

$$
\operatorname{Prob}_{a \in B(\bar{a}, \alpha)}\left\{\mathscr{C}(a)>\varepsilon^{-1}\right\}=\frac{\operatorname{vol}\left(T_{\text {dist }}(\Sigma, \varepsilon) \cap B(\bar{a}, \alpha)\right)}{\operatorname{vol}(B(\bar{a}, \alpha))} .
$$

Thus uniform smoothed analysis means to provide bounds on the volume of the intersection of $\varepsilon$-neighborhoods of $\Sigma$, relative to the distance function dist, with balls of radius $\alpha$. We note that for compact data spaces $\mathscr{D}$, uniform smoothed analysis interpolates transparently between worst-case and average-case analysis. Indeed, when $\alpha=0$ we get worst-case analysis, while for $\alpha=\operatorname{diam}(\mathscr{D})$ we obtain average-case analysis.

For conic condition numbers, this general concept specializes in the following way. The data space is the unit $m$-sphere $S^{m} \subset \mathbb{R}^{m+1}, \Sigma$ is a lower dimensional subset of $S^{m}$ such that $\Sigma=-\Sigma$ (in many applications it is an algebraic hypersurface) and the conic condition number $\mathscr{C}(a)$ of $a \in S^{m}$ is defined by (2) with respect to the distance function $\operatorname{dist}(a, b):=\sin d(a, b)$, where $d$ refers to the angular (i.e., Riemannian) distance in $S^{m}$. (We could as well consider the data space as the real projective space $\mathbb{P}^{m}$ on which dist defines a metric.)

For defining the GCC-condition number, one takes as the data space the $n$th power $\left(S^{m}\right)^{n}=S^{m} \times \cdots \times S^{m}$ of the sphere $S^{m}$ with the metric defined as $d(A, B):=\max _{1 \leq i \leq n} d\left(a_{i}, b_{i}\right)$, where $A=\left(a_{1}, \ldots, a_{n}\right), B=\left(b_{1}, \ldots, b_{n}\right)$ and $d\left(a_{i}, b_{i}\right)$ again denotes the angular distance in $S^{m}$. The distance function is defined by $\operatorname{dist}(A, B):=\sin d(A, B)$ and the GCC-condition number $\mathscr{C}(A)$ is given by $\mathscr{C}(A)=1 / \operatorname{dist}\left(A, \Sigma_{n, m}\right)$, where $\Sigma_{n, m} \subset\left(S^{m}\right)^{n}$ denotes the set of ill-posed inputs, which is a semialgebraic subset of codimension one (cf. $\S 2.3$ for details).

\subsection{Adversarial distributions}

An advantage of the uniform model is that, for conic condition numbers, results on smoothed analysis easily extend to more general families of probability distributions. To obtain such robustness results we rely on a general boosting technique developed in Hauser and Müller [18] and apply it similarly as in Cucker et al. [11]

The framework is the following (cf. $§ 2.4)$. Consider the spherical cap $B(\bar{a}, \alpha):=\left\{x \in S^{m} \mid d(x, a) \leq \alpha\right\}$ in the sphere $S^{m}$ centered at $\bar{a} \in S^{m}$ and having angular radius $\alpha \in(0, \pi / 2]$. Let $\nu$ denote the uniform measure on $B(\bar{a}, \alpha)$ and suppose that $\mu_{\bar{a}}$ is a $\nu$-absolutely continuous probability measure on $S^{m}$, say $\mu_{\bar{a}}(G)=\int_{G} f d \nu$ for measurable sets $G$. We further assume that 
the density $f$ is of the form $f(x)=g(\sin d(x, \bar{a}))$ with a monotonically decreasing function $g:[0, \sigma] \rightarrow[0, \infty]$ of the form

$$
g(r)=C r^{-\beta} h(r),
$$

where $\sigma:=\sin \alpha, \beta<m, h:[0, \sigma] \rightarrow[0, \infty)$ is a continuous function satisfying $h(0) \neq 0$, and $C=I_{m}(\alpha) / I_{m-\beta}(\alpha)$ is a normalizing constant, where $I_{k}(\alpha):=\int_{0}^{\alpha}(\sin t)^{k-1} d t$, cf. $\S 2.2$. Thus, the support of $\mu_{\bar{a}}$ is contained in $B(\bar{a}, \alpha)$ and the density of $\mu_{\bar{a}}$ is radially symmetric with a pole of order $\beta$ at $\bar{a}$. Such distributions were called adversarial in [11]. The exponent $\beta$ and the quantity $H:=\sup _{0 \leq r \leq \sigma} h(r)$ are the only parameters entering the bound below. In the special case $\beta=0$, the density of $\mu_{\bar{a}}$ does not have a singularity and the situation considerably simplifies: We have $C=1, g=h$ and the estimate $\mu_{\bar{a}}(B) \leq H \cdot \nu(B)$ holds for any measurable set $G$.

To extend this estimate to the general case, the smoothness parameter $s$ of a $\nu$-absolutely continuous distributions was defined in [18], and in [11, Lemma 3.2], it was shown that $s=1-\beta / m$. This means that $s$ is the largest number $s^{\prime}>0$ for which the following is true: For every $\varepsilon>0$ there exists $\delta(\varepsilon)>0$ such that $\nu(G) \leq \delta(\varepsilon)$ implies $\mu_{\bar{a}}(G) \leq \nu(B)^{s^{\prime}-\varepsilon}$ for all measurable sets $G$. This allows to obtain tail bounds for $\mu_{\bar{a}}$ from tail bounds for $\nu$.

Bürgisser et al. [7] provided a general smoothed analysis of conic condition numbers for uniform perturbations. This was recently extended by Cucker et al. [11] to the model of adversarial perturbations, who obtained the following robust smoothed analysis estimate.

Theorem 1.1 Let $\mathscr{C}$ be a conic condition number with set of ill-posed inputs $\Sigma \subseteq S^{m}$ and assume that $\Sigma$ is contained in an algebraic hypersurface of degree $d$. Then we have for a random perturbation from any adversarial distribution $\mu_{\bar{a}}$ on $B(\bar{a}, \alpha)$ with center $\bar{a} \in S^{m}$, angular radius $\alpha \in(0, \pi / 2]$ and parameters $\beta, H, \sigma=\sin \alpha$ that

$$
\mathbb{E}_{\mu_{\bar{a}}}(\ln \mathscr{C}) \leq \ln \frac{m^{2} d}{\sigma}+\frac{1}{1-\beta / m} \ln \frac{2 e H^{2} m}{\ln (\pi m / 2)}+\ln \frac{13 \pi}{2} .
$$

It is remarkable that the only problem dependent parameters entering the above bound are the dimension $m$ and the degree $d$. The only distribution dependent parameters entering the bound are $\sigma, \beta$, and $H$. This result has a wide range of applications to linear and polynomial equation solving. 


\subsection{Main results}

The goal of this paper is to prove the following analogue of Theorem 1.1 for the GCC-condition number of the linear programming feasibility problem.

Theorem 1.2 Let $\mathscr{C}$ denote the GCC condition number defined on $\left(S^{m}\right)^{n}$ and $n>m+1$. Suppose that $a_{i}$ is randomly chosen from an adversarial distribution $\mu_{\bar{a}_{i}}$ on $B\left(\bar{a}_{i}, \alpha\right)$ with center $\bar{a}_{i} \in S^{m}$, angular radius $\alpha \in(0, \pi / 2]$, and parameters $\beta, H, \sigma=\sin \alpha$, independently for $i=1, \ldots, n$. Then the random matrix $A$ with rows $a_{i}$ satisfies

$$
\mathbb{E}(\ln \mathscr{C}(A))=\mathcal{O}\left(\left(1-\frac{\beta}{m}\right)^{-2} \cdot \ln \frac{n H}{\sigma}\right) .
$$

In the case $\beta=0$, we have more precisely,

$$
\mathbb{E}(\ln \mathscr{C}(A)) \leq 12 \ln n+17 \ln m+6 \ln \frac{1}{\sigma}+8 \ln H+29=\mathcal{O}\left(\ln \frac{n H}{\sigma}\right) .
$$

As an application let $T(A)$ denote the number of iterations of the primaldual interior point method of [12] for solving the linear programming feasibility problem $\exists x \in \mathbb{R}^{m+1} A x<0$ (or its dual problem). Theorem 1.2 implies that

$$
\mathbb{E}(T(A))=\mathcal{O}\left(\left(1-\frac{\beta}{m}\right)^{-2} \cdot \sqrt{n} \cdot \ln \frac{n H}{\sigma}\right),
$$

for a random matrix $A$ with independent rows $a_{i}$ from a adversarial distribution $\mu_{\bar{a}_{i}}$ with parameters $\beta, H$.

For the uniform distribution on $S^{m}$, by essentially the same method, we can improve the estimates of Theorem 1.2 obtaining a result that was previously shown in [7] by a very different technique.

Corollary 1.3 Suppose that the rows of the matrix $A$ are independently chosen in $S^{m}$ according to the uniform distribution and $n>m+1$. Then

$$
\mathbb{E}(\ln \mathscr{C}(A))=\mathcal{O}(\ln m) .
$$

The paper is organized as follows. Section 2 is devoted to preliminaries on spherically convex sets, the GCC-condition number, and adversarial probability distributions. In Section 3 we state and prove probability tail bounds for the GCC-condition number under adversarial random perturbations and prove our main results. The proof essentially reduces the adversarial to the uniform case and then uses geometric arguments for the uniform case. A principal ingredient of the proof of the uniform case is an upper bound on 
the volume of the neighborhood of spherically convex sets (Theorem 3.3), that is stated in Section 3.1, but whose proof is deferred to Section 4. The proof of the latter result proceeds along the lines of [7] and uses some deeper results from integral and differential geometry (Weyl's tube formula, kinematic formula).

Acknowledgments. We thank Felipe Cucker and Martin Lotz for numerous helpful discussions. We are grateful to an anonymous referee for constructive criticism that led to more general results and a significantly better presentation of the paper.

\section{Preliminaries}

\subsection{Convex sets in spheres}

A general reference about convex sets is [30]. Glasauer's thesis [16] is a useful reference for the integral geometry of spherically convex sets.

A convex cone in $\mathbb{R}^{m+1}$ is a subset that is closed under addition and multiplication with nonnegative scalars. We denote by cone $(M)$ the convex cone generated by a subset $M \subseteq \mathbb{R}^{m+1}$. More specifically, the convex cone generated by points $a_{1}, \ldots, a_{k} \in \mathbb{R}^{m+1}$ is given by

$$
\text { cone }\left\{a_{1}, \ldots, a_{k}\right\}:=\left\{x \in \mathbb{R}^{m+1} \mid \exists \lambda_{1} \geq 0, \ldots, \lambda_{k} \geq 0 \quad x=\sum_{i=1}^{k} \lambda_{i} a_{i}\right\} .
$$

A convex cone $C$ is called pointed iff $C \cap(-C)=\{0\}$. It is known that $C$ is pointed iff $C \backslash\{0\}$ is contained in an open halfspace whose bounding hyperplane goes through the origin. Clearly, if $a_{i} \neq 0$ for $i=1, \ldots, k$, then cone $\left\{a_{1}, \ldots, a_{k}\right\}$ is pointed iff 0 is not contained in the convex hull $\operatorname{conv}\left\{a_{1}, \ldots, a_{k}\right\}$.

We use convex cones to define the notion of convexity for subsets of the sphere $S^{m}=\left\{x \in \mathbb{R}^{m+1} \mid\|x\|=1\right\}$. Let $x, y \in S^{m}$ be such that $x \neq \pm y$. We call $[x, y]:=$ cone $\{x, y\} \cap S^{m}$ the great circle segment connecting $x$ and $y$.

Definition 2.1 A subset $K$ of $S^{m}$ is called (spherically) convex iff we have $[x, y] \subseteq K$ for all $x, y \in K$ with $x \neq \pm y$. We call $K$ properly convex iff it is nonempty, convex, and does not contain a pair of antipodal points.

We denote by $\operatorname{sconv}(M):=\operatorname{cone}(M) \cap S^{m}$ the convex hull of a subset $M$ of $S^{m}$, which is the smallest convex set containing $M$. Clearly, $M$ is convex iff $M=\operatorname{sconv}(M)$. The closure of a convex set is convex as well. It is easy to see that a convex subset $K$ of $S^{m}$ is contained in a closed halfspace, unless $K=S^{m}$. A properly convex set $K$ is always contained in an open halfspace. 
Definition 2.2 The dual set of a subset $M \subseteq S^{m}$ is defined as

$$
\breve{M}:=\left\{a \in S^{m} \mid \forall x \in M\langle a, x\rangle \leq 0\right\} .
$$

Clearly, $\breve{M}$ is a closed convex set disjoint to $M$. The hyperplane separation theorem implies that the dual of $\breve{M}$ equals the closure of sconv $(M)$. We note that $M \subseteq N$ implies $\breve{M} \supseteq \breve{N}$. Finally, it is important that $\breve{M}$ has nonempty interior iff $M$ does not contain a pair of antipodal points, that is, "nonempty interior" and "properly convex" are dual properties.

By a convex body $K$ in $S^{m}$ we will understand a closed convex set $K$ such that both $K$ and $\breve{K}$ have nonempty interior, i.e., both are properly convex. The map $K \mapsto \breve{K}$ is an involution of the set of convex bodies in $S^{m}$.

\subsection{Distances, neighborhoods, and volumes}

We denote by $d(a, b) \in[0, \pi]$ the angular distance between two points $a, b$ on the sphere $S^{m}$. Clearly, this defines a metric on $S^{m}$. The (closed) ball of radius $\alpha \in[0, \pi]$ around $a \in S^{m}$ is defined as

$$
B(a, \alpha):=\left\{x \in S^{m} \mid d(x, a) \leq \alpha\right\}=\left\{x \in S^{m} \mid\langle a, x\rangle \geq \cos \alpha\right\} .
$$

This is the same as the spherical cap with center $a$ and angular radius $\alpha$. $B(a, \alpha)$ is convex iff $\alpha \leq \pi / 2$ or $\alpha=\pi$. In the case $\alpha \leq \pi / 2$, the dual set of $B(a, \alpha)$ equals $B(-a, \pi / 2-\alpha)$.

For a nonempty subset $M$ of $S^{m}$ we define the distance of $a \in S^{m}$ to $M$ as $d(a, M):=\inf \{d(a, x) \mid x \in M\}$. The dual set of $M$ can be characterized in terms of distances by: $a \in \breve{M} \Longleftrightarrow d(a, M) \geq \pi / 2$.

Lemma 2.3 Let $K$ be a convex body in $S^{m}$ and $a \in S^{m} \backslash(K \cup \breve{K})$. Then $d(a, K)+d(a, \breve{K})=\pi / 2$.

Proof. Let $b \in K$ such that $\varphi:=d(a, b)=d(a, K)$. Since $a \notin \breve{K}$ we have $\varphi<\pi / 2$. The point $b^{*}:=\langle a, b\rangle b$ is therefore nonzero and contained in $C:=$ cone $(K)$. Put $p^{*}:=a-b^{*}$. Then $\left\langle p^{*}, b\right\rangle=0,\left\langle p^{*}, a\right\rangle=\sin ^{2} \varphi$, and $\left\langle p^{*}, p^{*}\right\rangle=\sin ^{2} \varphi$. In particular $p^{*} \neq 0$.

By construction, $b^{*}$ is the point of $C$ closest to $a$. It follows that $\{x \in$ $\left.\mathbb{R}^{m+1} \mid\left\langle p^{*}, x\right\rangle=0\right\}$ is a supporting hyperplane of $C$. Hence $\left\langle p^{*}, x\right\rangle \leq 0$ for all $x \in C$ and the point $p:=p^{*} /\left\|p^{*}\right\|$ therefore belongs to $K$. Moreover, $\langle p, a\rangle=\sin \varphi$, which implies $d(a, p)=\pi / 2-\varphi$. Hence

$$
d(a, K)+d(a, \breve{K}) \leq d(a, b)+d(a, p)=\pi / 2 .
$$


To complete the proof it suffices to show that $d(a, \breve{K})=d(a, p)$. Suppose there exists $p^{\prime} \in \breve{K}$ such that $d\left(a, p^{\prime}\right)<d(a, p)$. Then $d\left(b, p^{\prime}\right) \leq d(b, a)+$ $d\left(a, p^{\prime}\right)<d(b, a)+d(a, p)=\pi / 2$ which contradicts the fact that $b \in \breve{K}$.

Sometimes it will be useful to consider the projective distance between two points $a, b \in S^{m}$, which is defined as $d_{\mathbb{P}}(a, b):=\sin d(a, b)$. It is straightforward to check that $d_{\mathbb{P}}$ satisfies the triangle inequality. However, it is not a metric on $S^{m}$, as $d_{\mathbb{P}}(a, b)=0$ iff $a= \pm b$. Hence the ball of radius $\sin \alpha$, measured with respect to the projective distance, equals $B(a, \alpha) \cup B(-a, \alpha)$. We denote this set suggestively by $B( \pm a, \alpha)$ and call it the projective ball with center $\pm a$ and radius $\alpha$.

For $0 \leq \varphi \leq \pi / 2$, the $\varphi$-neighborhood of a nonempty subset $M$ of $S^{m}$ is defined as $T(M, \varphi):=\left\{x \in S^{m} \mid d(x, M)<\varphi\right\}$. If $M$ is the boundary $\partial K$ of a properly convex set $K$ in $S^{m}$, we call

$$
T_{o}(\partial K, \varphi):=T(\partial K, \varphi) \backslash K \quad \text { and } \quad T_{i}(\partial K, \varphi):=T(\partial K, \varphi) \cap K
$$

the outer $\varphi$-neighborhood and inner $\varphi$-neighborhood of $\partial K$, respectively. Clearly, we have $T(\partial K, \varphi)=T_{o}(\partial K, \varphi) \cup T_{i}(\partial K, \varphi)$.

In order to compute the $m$-dimensional volume of such neighborhoods, the following functions $J_{m, k}(\alpha)$ are relevant:

$$
J_{m, k}(\alpha):=\int_{0}^{\alpha}(\sin \rho)^{k-1}(\cos \rho)^{m-k} d \rho \quad(1 \leq k \leq m) .
$$

Recall that $\mathcal{O}_{m}:=\operatorname{vol} S^{m}=2 \pi^{(m+1) / 2} / \Gamma((m+1) / 2)$ equals the $m$-dimensional volume of $S^{m}$. It is known that vol $T\left(S^{m-k}, \alpha\right)=\mathcal{O}_{m-k} \mathcal{O}_{k-1} J_{m, k}(\alpha)$. Some estimations of these volumes can be found in [7, Lemmas 2.1-2.2].

\subsection{The GCC condition number}

We study the problem of deciding for a given instance $A \in \mathbb{R}^{n \times(m+1)}$ whether there exists a nonzero solution $x \in \mathbb{R}^{m+1} \backslash\{0\}$ such that $A x \leq 0$. In the following we assume that $n>m+1$. (The other case is considerably less interesting.) Without loss of generality we may assume that the row vectors $a_{i}$ have euclidean length one, and hence interpret $A=\left(a_{1}, \ldots, a_{n}\right)$ as an element of the product $\left(S^{m}\right)^{n}$ of spheres.

We write $\operatorname{sconv}(A):=\operatorname{sconv}\left\{a_{1}, \ldots, a_{n}\right\}$ for the convex hull of the given points. The set of solutions in $S^{m}$ of the system of inequalities $A x \leq 0$ equals the dual set of $\operatorname{sconv}(A)$. 
Definition 2.4 An instance $A \in\left(S^{m}\right)^{n}$ is called feasible iff its set of solutions is nonempty, otherwise $A$ is called infeasible. An instance $A$ is called strictly feasible iff its set of solutions has nonempty interior. We denote by $\mathcal{F}_{n, m}$ and $\mathcal{F}_{n, m}^{\circ}$ the set of feasible and strictly feasible instances, respectively. The set of ill-posed instances is defined as $\Sigma_{n, m}:=\mathcal{F}_{n, m} \backslash \mathcal{F}_{n, m}^{\circ}$. The set of infeasible instances is denoted by $\mathcal{I}_{n, m}$.

Remark 2.5 An instance $A \in\left(S^{m}\right)^{n}$ is strictly feasible iff sconv $(A)$ is properly convex, that is, cone $(A)$ is pointed. Furthermore, a feasible instance $A$ is ill-posed iff 0 is contained in the euclidean convex hull of $a_{1}, \ldots, a_{n}$ (cf. [5, Lemma 3.2]).

We remark that $\mathcal{F}_{n, m}$ is a compact subset of $\left(S^{m}\right)^{n}$ with nonempty interior $\mathcal{F}_{n, m}^{\circ}$ and topological boundary $\Sigma_{n, m}$. Moreover, $\mathcal{I}_{n, m}$ is nonempty and $\Sigma_{n, m}$ is also the topological boundary of $\mathcal{I}_{n, m}$. (Here we use $n>m+1$.)

We define a metric on $\left(S^{m}\right)^{n}$ by setting for $A, B \in\left(S^{m}\right)^{n}$ with components $a_{i}, b_{i} \in S^{m}$

$$
d(A, B):=\max _{1 \leq i \leq n} d\left(a_{i}, b_{i}\right) .
$$

The distance of $A$ to a nonempty subset $M \subseteq\left(S^{m}\right)^{n}$ is defined as $d(A, M):=$ $\inf \{d(A, B) \mid B \in M\}$. We denote by $B(\bar{A}, \alpha):=\left\{A \in\left(S^{m}\right)^{n} \mid d(A, \bar{A}) \leq \alpha\right\}$ the closed ball with center $\bar{A}$ and radius $\alpha$. Clearly, this is the product of the balls $B\left(\bar{a}_{i}, \alpha\right)$ for $i=1, \ldots, n$.

The following definition is due to Goffin [17] and Cheung and Cucker [8].

Definition 2.6 The GCC condition number of $A \in\left(S^{m}\right)^{n}$ is defined as $\mathscr{C}(A)=1 / \sin d\left(A, \Sigma_{n, m}\right)$.

This condition number can be characterized in a more explicit way.

Definition 2.7 A smallest including cap (SIC) for $A \in\left(S^{m}\right)^{n}$ is a spherical cap of minimal radius containing the points $a_{1}, \ldots, a_{n}$.

We remark that by a compactness argument, a SIC always exists. It can be shown that a SIC is unique if $A$ is strictly feasible. However, for infeasible $A$, there may be several SICs (consider for instance three equidistant points on the circle). We denote the radius of a SIC of $A$ by $\rho(A)$. An instance $A$ is strictly feasible iff $\rho(A)<\pi / 2$. For more information on this we refer to $[10,5]$.

The following result is due to Cheung and Cucker [8]. This characterization is essential for any probabilistic analysis of the GCC condition number. 
Theorem 2.8 We have

$$
d\left(A, \Sigma_{n, m}\right)= \begin{cases}\frac{\pi}{2}-\rho(A) & \text { if } A \in \mathcal{F}_{n, m}, \\ \rho(A)-\frac{\pi}{2} & \text { if } A \in\left(S^{m}\right)^{n} \backslash \mathcal{F}_{n, m} .\end{cases}
$$

In particular, $d\left(A, \Sigma_{n, m}\right) \leq \frac{\pi}{2}$ and $\mathscr{C}(A)^{-1}=|\cos \rho(A)|$.

\subsection{Adversarial probability distributions}

The proof of our robustness result relies on a general boosting technique developed in [18], that allows to extend probability tail bounds obtained for one fixed distribution to larger classes of distributions. We explain this technique in our situation of interest.

Let $\nu:=\nu_{\bar{a}, \sigma}$ denote the uniform distribution on the spherical disk $B(\bar{a}, \alpha)$, where $\alpha \in\left(0, \frac{\pi}{2}\right]$ and $\sigma:=\sin \alpha$. We assume that $\mu$ is a $\nu$-absolutely continuous probability measure, i.e., it can be written with a density $f$ as $\mu(G)=\int_{G} f d \nu$ for Borel measurable sets $G$. In certain cases, it is possible to bound $\mu(G)$ in terms of $\nu(G)$ if the latter is sufficiently small. This is done with the smoothness parameter $s$ of $\mu$ with respect to $\nu$, which is defined as $s:=\lim _{\delta \rightarrow 0} \inf \delta$, where we have set for $\delta \in(0,1)$, using the convention $\ln 0:=-\infty$,

$$
\inf \delta:=\inf \left\{\frac{\ln \mu(G)}{\ln \nu(G)} \mid 0<\nu(G) \leq \delta\right\} .
$$

If $s$ is positive, we say that $\mu$ is uniformly $\nu$-absolutely continuous. In this case, it is easy to see that $s$ is the largest nonnegative real number $s^{\prime}$ with the property that for all $\varepsilon>0$ there exists a tolerance $\delta(\varepsilon)>0$ such that $\nu(G) \leq \delta(\varepsilon)$ implies $\mu(G) \leq \nu(G)^{s^{\prime}-\varepsilon}$ for all $G$, cf. [18].

We will apply this framework to a specific class of distributions $\mu$. Not only will it be important to know the smoothness parameter, but also to explicitly compute bounds for the tolerance $\delta(\varepsilon)$.

An adversarial probability distribution $\mu_{\bar{a}}$, for $\bar{a} \in S^{m}$, was defined in [11] as a $\nu$-absolutely continuous measure given by $\mu_{\bar{a}}(G)=\int_{G} f d \nu$, where again $\nu$ denotes the uniform distribution on $B(\bar{a}, \alpha)$. We further require that the density $f$ is of the form $f(x)=g(\sin d(x, \bar{a}))$ with a monotonically decreasing function $g:[0, \sigma] \rightarrow[0, \infty]$ given by

$$
g(r)=C r^{-\beta} h(r),
$$

where $0 \leq \beta<m$, and $h:[0, \sigma] \rightarrow[0, \infty)$ is a continuous function satisfying $h(0) \neq 0$. Thus, the support of $\mu_{\bar{a}}$ is contained in $B(\bar{a}, \alpha)$ and the density of $\mu_{\bar{a}}$ is radially symmetric with a pole of order $\beta$ at $\bar{a}$. Clearly, $\bar{a}$ is the 
mean of $a$ with respect to $\mu_{\bar{a}}$. It is convenient to assume the normalization $C:=I_{m}(\alpha) / I_{m-\beta}(\alpha)$, where

$$
I_{k}(\alpha):=J_{k, k}(\alpha)=\int_{0}^{\alpha}(\sin t)^{k-1} d t
$$

compare (4) (we slightly deviate here from the notation in [11]). Then $\mu_{\bar{a}}$ is a probability distribution iff

$$
I_{m-\beta}(\alpha)=\int_{0}^{\alpha}(\sin t)^{m-\beta-1} h(\sin t) d t .
$$

The maximum of $h$ is denoted by $H:=\sup _{0 \leq r \leq \sigma} h(r)$, which is easily seen to satisfy $H \geq 1$. We note that the uniform distribution on $B(\bar{a}, \alpha)$ is obtained by choosing $\beta=0, C=1$ and for $g=h$ the function identically equal to 1 .

The following technical lemma is an immediate consequence of $[11$, Lemmas 3.2-3.3, Equation (3.1)].

Lemma 2.9 1. The smoothness parameter of $\mu$ with respect to $\nu$ equals $s=1-\beta / m$.

2. For $c:=\frac{1}{2} s=\frac{1}{2}\left(1-\frac{\beta}{m}\right) \leq \frac{1}{2}$ and the tolerance $\delta_{c}$ defined by

$$
\delta_{c}:=\frac{2}{\pi m}\left(\frac{1}{H} \cdot \sqrt{1-\left(\frac{2}{\pi m}\right)^{\frac{1}{m}}}\right)^{1 / c}
$$

we have for all measurable $G \subseteq S^{m}$,

$$
\nu(G) \leq \delta_{c} \Longrightarrow \mu_{\bar{a}}(G) \leq(\nu(G))^{c} .
$$

Remark 2.10 In the case $\beta=0$ where the density of $\mu_{\bar{a}}$ has no singularity the situation simplifies. Clearly, $\mu_{\bar{a}}(G) \leq H \nu(G)$ for all $G$. This directly implies that the smoothness parameter equals 1 . Moreover, the tolerance $\delta=\frac{1}{H^{2}}$ is sufficient for the implication $\nu(G) \leq \delta \Rightarrow \mu \bar{a}(G) \leq \nu(G)^{\frac{1}{2}}$.

Since we will work in the product of spheres $\left(S^{m}\right)^{n}$ we define on it the adversarial distributions $\mu_{\bar{A}}$ with center $\bar{A}=\left(\bar{a}_{1}, \ldots, \bar{a}_{n}\right) \in\left(S^{m}\right)^{n}$ by taking the product measure $\mu_{\bar{A}}:=\mu_{\bar{a}_{1}} \times \ldots \times \mu_{\bar{a}_{n}}$. This is a probability distribution whose support is contained in the product of caps $B(\bar{A}, \alpha):=$ $B\left(\bar{a}_{1}, \alpha\right) \times \ldots \times B\left(\bar{a}_{n}, \alpha\right)$. 


\section{Robust smoothed analysis of $\mathscr{C}(A)$}

The goal of this section is to provide smoothed analysis estimates for the condition number $\mathscr{C}(A)$ in the model where $A=\left(a_{1}, \ldots, a_{n}\right)$ is chosen at random according to an adversarial distribution $\mu_{\bar{A}}$. The center $\bar{A} \in\left(S^{m}\right)^{n}$ of the perturbation is arbitrary. Recall that $\mathcal{F}_{n, m}$ and $\mathcal{I}_{n, m}$ denote the sets of feasible and infeasible instances $A \in\left(S^{m}\right)^{n}$, respectively.

Theorem 3.1 Let $\alpha \in(0, \pi / 2], \sigma=\sin \alpha$, and $\bar{A} \in\left(S^{m}\right)^{n}$, where $n>m+1$. Assume that $A \in B(\bar{A}, \alpha)$ is chosen at random according to an adversarial distribution $\mu_{\bar{A}}$. Let the exponent $c=\frac{1}{2}(1-\beta / m)$ and the tolerance $\delta_{c}$ be defined as in Lemma 2.9. Then we have

$$
\operatorname{Prob}\left\{A \in \mathcal{F}_{n, m} \text { and } \mathscr{C}(A) \geq t\right\} \leq n\left(\frac{13 m(m+1)}{2 \sigma}\right)^{c} t^{-c} .
$$

provided $t \geq \frac{13 m(m+1)}{2 \sigma \delta_{c}}$. Moreover, we have for $t \geq 1$,

$$
\operatorname{Prob}\left\{A \in \mathcal{I}_{n, m} \text { and } \mathscr{C}(A) \geq t\right\} \leq n\left(\frac{1690 m^{2}(m+1)}{4 \sigma^{2}}\right)^{c} t^{-c}\left(\delta_{c}^{-c}+c n \ln t\right) .
$$

Remark 3.2 For the uniform distribution $(\beta=0, h \equiv 1)$ the bounds in $(\mathrm{F})$ and (I) can easily be improved by avoiding the use of Lemma 2.9. In particular, on the right-hand side of (F) and (I) one gets $t^{-1}$ and $t^{-1} \ln t$ instead of $t^{-1 / 2}$ and $t^{-1 / 2} \ln t$.

The overall strategy of the proof of Theorem 3.1 is the same as in [15]. However, the crucial component in [15], namely a result due to Ball [2], is substituted by Corollary 3.4, which is stated in the next section.

\subsection{A bound on the volume of convex neighborhoods}

We state here an upper bound on the volume of the intersection of neighborhoods of spherically convex sets with spherical caps.

Theorem 3.3 Let $K$ be a properly convex subset of $S^{m}$, let $a \in S^{m}$, and $0<\alpha, \varphi \leq \pi / 2$. Then, writing $\sigma=\sin \alpha$ and $\varepsilon=\sin \varphi$, we have the following upper bound for the volume of the outer neighborhood of $\partial K$ :

$\frac{\operatorname{vol}\left(T_{o}(\partial K, \varphi) \cap B( \pm a, \alpha)\right)}{\operatorname{vol} B( \pm a, \alpha)} \leq \sum_{k=1}^{m-1}\left(\begin{array}{c}m \\ k\end{array}\right)\left(1+\frac{\varepsilon}{\sigma}\right)^{m-k}\left(\frac{\varepsilon}{\sigma}\right)^{k}+\frac{m \mathcal{O}_{m}}{2 \mathcal{O}_{m-1}}\left(\frac{\varepsilon}{\sigma}\right)^{m}$. 
The same upper bound holds for the volume $\frac{\operatorname{vol}\left(T_{i}(\partial K, \varphi) \cap B( \pm a, \alpha)\right)}{\operatorname{vol} B( \pm a, \alpha)}$ of the inner neighborhood of $\partial K$.

The main result of $[7$, Theorem 1.2] gives a bound on the volume of the intersection of a projective ball $B( \pm a, \alpha)=B(a, \alpha) \cup B(-a, \alpha)$ with the $\varphi$ neighborhood of a real algebraic hypersurface in the sphere $S^{m}$, given as the zero set of polynomial of degree $d$. The above theorem says that essentially the same volume bound holds for the boundary of a properly convex set $K$ in $S^{m}$, if we formally replace in this bound the degree $d$ by $1 / 2$.

The proof of Theorem 3.3, which is quite involved, is deferred to Section 4. By essentially the same argument as in the proof of [7, Prop. 3.5] one can derive from Theorem 3.3 the following corollary.

Corollary 3.4 Under the assumptions of Theorem 3.3 we have the following upper bound for the volume of the outer neighborhood of $\partial K$ :

$$
\frac{\operatorname{vol}\left(T_{o}(\partial K, \varphi) \cap B( \pm a, \alpha)\right)}{\operatorname{vol} B( \pm a, \alpha)} \leq \frac{13 m}{4} \frac{\varepsilon}{\sigma} \quad \text { if } \varepsilon \leq \frac{\sigma}{2 m} .
$$

The same upper bound holds for the relative volume of the inner neighborhood of $\partial K$.

\subsection{Two auxiliary results}

The proofs of the following two results are similar as in Dunagan et al. [15]. We use the notation $[n]:=\{1,2, \ldots, n\}$ for $n \in \mathbb{N}$.

Proposition 3.5 Let $A=\left(a_{1}, \ldots, a_{n}\right) \in \mathcal{F}_{n, m}^{\circ}, 0<\varphi \leq \pi / 2$, and $\varepsilon=\sin \varphi$. If $\mathscr{C}(A) \geq(m+1) \varepsilon^{-1}$, then there exists $i \in[n]$ such that

$$
a_{i} \in T\left(\partial K_{i}, \varphi\right) \backslash K_{i}
$$

where $K_{i}:=-\operatorname{sconv}\left\{a_{1}, \ldots, a_{i-1}, a_{i+1}, \ldots, a_{n}\right\}$.

Proof. There exists $q \in \operatorname{sconv}(A)$ such that $\left\langle a_{i}, q\right\rangle>0$ for all $i \in[n]$. Indeed, if $q$ is taken as the center of the SIC of $A$ then this follows from [10, Lemma 4.5] (see also [5, Lemma 3.2]).

We note that $a_{i} \notin K_{i}$ for all $i \in[n]$. Otherwise $0 \in \operatorname{conv}\left\{a_{1}, \ldots, a_{n}\right\}$, hence $A \in \Sigma_{n, m}$, which contradicts our assumption that $A$ is strictly feasible. It follows that $d\left(a_{i}, \partial K_{i}\right)=d\left(a_{i}, K_{i}\right)$.

We assume now $d\left(a_{i}, K_{i}\right)>\varphi$ for all $i \in[n]$. Our goal is to show that $\sin d\left(A, \Sigma_{n, m}\right)>\frac{1}{m+1} \varepsilon$. Then we are done, since $\mathscr{C}(A)^{-1}=\sin d\left(A, \Sigma_{n, m}\right)$ by Definition 2.6. 
We proceed now similarly as in [15, Lemma 2.3.10]. By continuity we assume w.l.o.g. that $\varphi<\pi / 2$. We distinguish two cases. If $a_{i} \notin \breve{K}_{i}$, then Lemma 2.3 tells us that $d\left(a_{i}, K_{i}\right)+d\left(a_{i}, \breve{K}_{i}\right)=\pi / 2$. Hence $d\left(a_{i}, \breve{K}_{i}\right)<$ $\pi / 2-\varphi$. Choose $p_{i} \in \operatorname{int}\left(\breve{K}_{i}\right)$ such that $d\left(a_{i}, p_{i}\right)<\pi / 2-\varphi$. This implies $\left\langle a_{i}, p_{i}\right\rangle>\cos (\pi / 2-\varphi)=\varepsilon$. In the case $a_{i} \in \breve{K}_{i}$ we take any $p_{i} \in \operatorname{int}\left(\breve{K}_{i}\right)$ close enough to $a_{i}$ such that $\left\langle a_{i}, p_{i}\right\rangle>\varepsilon$.

In both cases we have achieved the following

$$
\left\langle a_{i}, p_{i}\right\rangle>\varepsilon \text { and } \forall j \neq i\left\langle a_{j}, p_{i}\right\rangle>0 .
$$

This implies for all $i$ that $\left\langle p_{i}, q\right\rangle>0$, as $q \in$ cone $\left\{a_{1}, \ldots, a_{n}\right\}$.

Consider now for $i \in[n]$ the following convex sets in $S^{m}$

$$
C_{i}:=\left\{x \in S^{m} \mid\left\langle a_{i}, x\right\rangle>\frac{\varepsilon}{m+1} \text { and }\langle x, q\rangle>0\right\}
$$

containing $p_{i}$. We claim that the intersection of any $m+1$ of these sets is nonempty. Indeed, let $I \subseteq[n]$ be of cardinality $m+1$ and consider $p^{*}:=\frac{1}{m+1} \sum_{j \in I} p_{j}$. Note that $\left\|p^{*}\right\| \leq 1$. We obtain for any $i \in I$, using (5),

$$
\left\langle a_{i}, p^{*}\right\rangle=\frac{1}{m+1} \sum_{j \in I}\left\langle a_{i}, p_{j}\right\rangle \geq \frac{1}{m+1}\left\langle a_{i}, p_{i}\right\rangle>\frac{\varepsilon}{m+1} .
$$

Moreover, $\left\langle p^{*}, q\right\rangle>0$, hence $p^{*} \neq 0$. It follows that $p:=p^{*} /\left\|p^{*}\right\|$ is contained in $C_{i}$ for any $i \in I$, which shows the claim.

Consider the affine hyperplane $E:=\left\{x \in \mathbb{R}^{m+1} \mid\langle x, q\rangle=1\right\}$ of dimension $m$ and the perspective map

$$
\pi:\left\{x \in S^{m} \mid\langle x, q\rangle>0\right\} \rightarrow E, x \mapsto\langle q, x\rangle^{-1} x .
$$

Then the $\pi\left(C_{i}\right)$ are convex subsets of $E$, with the property that any $m+1$ of these have a nonempty intersection. Helly's theorem [30] implies that $\pi\left(C_{1}\right) \cap \cdots \cap \pi\left(C_{n}\right)$ is nonempty. Hence there is a point $a \in \bigcap_{i=1}^{n} C_{i}$. We have $d\left(a_{i}, a\right)<\alpha:=\arccos \left((m+1)^{-1} \varepsilon\right)$ for all $i \in[n]$. Hence the spherical cap $B(a, \alpha)$ strictly contains all $a_{i}$. The radius $\rho(A)$ of the SIC of $A$ is therefore strictly smaller than $\alpha$. Hence, by Theorem $2.8, \sin d\left(A, \Sigma_{n, m}\right)=$ $\cos \rho(A)>\cos \alpha=(m+1)^{-1} \varepsilon$, as claimed.

The next proposition on the transition from the feasible to the infeasible case is similar as [15, Lemma 2.3.14].

Proposition 3.6 Let $A=\left(a_{1}, \ldots, a_{n}\right) \in \mathcal{F}_{n, m}$ and $K:=-\operatorname{sconv}(A)$. If $b \in K$, then $(A, b):=\left(a_{1}, \ldots, a_{n}, b\right)$ is infeasible or ill-posed and we have

$$
\mathscr{C}(A, b) \sin d(b, \partial K) \leq 10 \mathscr{C}(A) .
$$


Proof. W.l.o.g. $A$ is strictly feasible. The set of solutions

$$
C:=\left\{x \in S^{m} \mid\left\langle a_{1}, x\right\rangle \leq 0, \ldots,\left\langle a_{n}, x\right\rangle \leq 0\right\}
$$

is the dual of $\operatorname{sconv}(A)$. Hence $\breve{C}=\operatorname{sconv}(A)$. This means that $a \in K$ iff $\langle a, x\rangle \geq 0$ for all $x \in C$. Therefore, we have for all $a \in S^{m}$,

$$
a \notin K \Longleftrightarrow \exists x \in C\langle a, x\rangle<0 \Longleftrightarrow\left(a_{1}, \ldots, a_{n}, a\right) \text { is strictly feasible, }
$$

where the second equivalence follows from the assumption that $C$ has nonempty interior. A similar argument shows that $\left(a_{1}, \ldots, a_{n}, a\right)$ is ill-posed iff $a \in \partial K$. Therefore, we have

$$
d(b, \partial K)=\min \left\{d(b, a) \mid a \in S^{m} \text { such that }\left(a_{1}, \ldots, a_{n}, a\right) \in \Sigma_{n+1, m}\right\} .
$$

For proving the proposition we can assume without loss of generality that $b \in K \backslash \partial K$. Then $(A, b)$ is not strictly feasible by (6). Moreover, since $b \notin \partial K,(A, b)$ is not ill-posed. Hence $(A, b)$ is infeasible. We put now $\omega:=\sin d(b, \partial K)$ and claim that

$$
\omega \leq \min _{x \in C}\langle b, x\rangle
$$

In order to show this, suppose $q \in C$. The equivalence (6) and $b \in K$ imply that $\cos \theta:=\langle b, q\rangle \geq 0$. W.l.o.g. we may assume that $\|b-q \cos \theta\|^{2}=$ $1-\cos ^{2} \theta$ is positive (otherwise $\theta=0, b=q$, and $\langle b, q\rangle=1 \geq \omega$ ). It therefore makes sense to define $b^{\prime}:=(b-q \cos \theta) /\|b-q \cos \theta\|$. Then $b^{\prime} \in S^{m}$ and $\left\langle b^{\prime}, q\right\rangle=0$. Note that $d\left(b, b^{\prime}\right)=\pi / 2-\theta$. Therefore $\left(a_{1}, \ldots, a_{n}, b^{\prime}\right)$ is feasible. It is either strictly feasible, in which case $b^{\prime} \notin K$, or ill-posed, in which case $b^{\prime} \in \partial K$ (use (6)). Since $b \in K$ we conclude that $d(b, \partial K) \leq d\left(b, b^{\prime}\right)=$ $\pi / 2-\theta$. This implies

$$
\omega=\sin d(b, \partial K) \leq \cos \theta=\langle b, q\rangle
$$

and hence the claimed inequality (7). Moreover note that $d(b, \partial K) \leq \pi / 2$ and $\omega>0$ as $b \notin \partial K$.

Suppose now that $B(p, \rho)$ is the SIC for $A$. Since we assume $A$ to be strictly feasible $t:=\cos \rho$ is positive. By the characterization of the GCC condition number in Theorem 2.8 we have $t=\sin d\left(A, \Sigma_{n, m}\right)=\mathscr{C}(A)^{-1}$.

Put $\varphi:=\arcsin \left(\frac{1}{10} t \omega\right)$. For proving the proposition, it is enough to show the implication

$$
\forall\left(A^{\prime}, b^{\prime}\right) \in\left(S^{m}\right)^{n+1} \quad d\left(\left(A^{\prime}, b^{\prime}\right),(A, b)\right) \leq \varphi \Longrightarrow\left(A^{\prime}, b^{\prime}\right) \text { infeasible. }
$$


Indeed, this implies (using $d\left((A, b), \Sigma_{n+1, m}\right) \leq \pi / 2$, cf. Theorem 2.8)

$$
\mathscr{C}(A, b)^{-1}=\sin d\left((A, b), \Sigma_{n+1, m}\right) \geq \sin \varphi=\frac{1}{10} t \omega=\frac{1}{10} \mathscr{C}(A)^{-1} \omega,
$$

as claimed in the proposition.

We argue by contradiction. Suppose there is a feasible $\left(A^{\prime}, b^{\prime}\right)$ having distance at most $\varphi$ from $(A, b)$. Then there exists $x^{\prime} \in S^{m}$ such that

$$
\left\langle a_{1}^{\prime}, x^{\prime}\right\rangle \leq 0, \ldots,\left\langle a_{n}^{\prime}, x^{\prime}\right\rangle \leq 0,\left\langle b^{\prime}, x^{\prime}\right\rangle \leq 0 .
$$

Taking into account that $d\left(a_{i}^{\prime}, a_{i}\right) \leq \varphi$, we see that $d\left(a_{i}, x^{\prime}\right) \geq \pi / 2-\varphi$ and hence $\left\langle a_{i}, x^{\prime}\right\rangle \leq \sin \varphi$.

We put now $\tilde{x}:=x^{\prime}-\lambda p$ with $\lambda:=t^{-1} \sin \varphi$. As $\left\langle a_{i}, p\right\rangle \geq t$, we have for $i \in[n]$

$$
\left\langle a_{i}, \tilde{x}\right\rangle=\left\langle a_{i}, x^{\prime}\right\rangle-\lambda\left\langle a_{i}, p\right\rangle \leq \sin \varphi-\lambda t=0 .
$$

Note that $\tilde{x} \neq 0$ (otherwise $t=\sin \varphi$, which is impossible). Therefore, $\tilde{x} /\|\tilde{x}\|$ is well-defined and lies in $C$. Inequality (7) implies that $\omega\|\tilde{x}\| \leq\langle b, \tilde{x}\rangle$.

Put $\Delta b:=b^{\prime}-b$. Then $\|\Delta b\| \leq 2 \sin (\varphi / 2)$ by our assumption $d\left(b^{\prime}, b\right) \leq \varphi$. We obtain

$$
\begin{aligned}
\langle b, \tilde{x}\rangle & =\left\langle b^{\prime}-\Delta b, x^{\prime}-\lambda p\right\rangle=\left\langle b^{\prime}, x^{\prime}\right\rangle-\left\langle\Delta b, x^{\prime}\right\rangle-\left\langle b^{\prime}, \lambda p\right\rangle+\langle\Delta b, \lambda p\rangle \\
& \leq 0+\|\Delta b\|+\lambda+\|\Delta b\| \lambda .
\end{aligned}
$$

To arrive at a contradiction it is enough to verify that

$$
\|\Delta b\|+\lambda+\|\Delta b\| \lambda<\omega\|\tilde{x}\| .
$$

Note that $\|\tilde{x}\| \geq 1-\lambda,\|\Delta b\| \leq 2$, and $\omega \leq 1$. It is therefore sufficient to check that

$$
\|\Delta b\|+\lambda+2 \lambda<\omega-\lambda,
$$

that is,

$$
\|\Delta b\|+4 \lambda<\omega .
$$

Using $\sin \varphi=2 \sin (\varphi / 2) \cos (\varphi / 2)$ we get $\lambda=t^{-1} \sin \varphi \leq 2 t^{-1} \sin (\varphi / 2)$. It is therefore sufficient to show that

$$
2 \sin \frac{\varphi}{2}+8 t^{-1} \sin \frac{\varphi}{2}<\omega
$$

which is equivalent to

$$
(t+4) \sin \frac{\varphi}{2}<\frac{1}{2} t \omega
$$

As $t \leq 1$, it is enough to show that $5 \sin \frac{\varphi}{2}<\frac{1}{2} t \omega$. This is true, since by our assumption $\sin \frac{\varphi}{2}<\sin \varphi=\frac{1}{10} t \omega$. 


\subsection{Feasible instances}

We provide here the proof of the part of Theorem 3.1 dealing with feasible instances. That is, we wish to show the claimed bound $(\mathrm{F})$.

Let $\bar{A} \in\left(S^{m}\right)^{n}, 0<\alpha \leq \pi / 2, \sigma=\sin \alpha$, and $t \geq 13 m(m+1)\left(2 \sigma \delta_{c}\right)^{-1}$. Put $\varepsilon:=(m+1) t^{-1}$ and $\varphi:=\arcsin \varepsilon$. We suppose that $A \in\left(S^{m}\right)^{n}$ is chosen at random according to a distribution $\mu_{\bar{A}}$ on $\left(S^{m}\right)^{n}$ as defined in Section 2.4. Using Proposition 3.5 and the notation introduced there, we have

$\operatorname{Prob}\left\{A \in \mathcal{F}_{n, m}^{\circ}\right.$ and $\left.\mathscr{C}(A) \geq t\right\} \leq \sum_{i=1}^{n} \operatorname{Prob}\left\{A \in \mathcal{F}_{n, m}^{\circ}\right.$ and $\left.a_{i} \in T_{o}\left(\partial K_{i}, \varphi\right)\right\}$.

We first bound the probability on the right-hand side for $i=n$ by expressing it as an integral over $A^{\prime}:=\left(a_{1}, \ldots, a_{n-1}\right)$ of probabilities conditioned on $A^{\prime}$. Note that $\mu_{\bar{A}}=\mu_{\bar{A}^{\prime}} \times \mu_{\bar{a}_{n}}$ where $\bar{A}^{\prime}:=\left(\bar{a}_{1}, \ldots, \bar{a}_{n-1}\right)$. Moroever, $A \in \mathcal{F}_{n, m}^{\circ}$ iff $A^{\prime} \in \mathcal{F}_{n-1, m}^{\circ}$ and $a_{n} \notin K_{n}$, where we set now $K_{A^{\prime}}:=K_{n}=-\operatorname{sconv}\left(A^{\prime}\right)$, see (6). This implies

$$
\begin{aligned}
& \underset{\mu_{\bar{A}}}{\operatorname{Prob}}\left\{A \in \mathcal{F}_{n, m}^{\circ} \text { and } a_{n} \in T_{o}\left(\partial K_{n}, \varphi\right)\right\} \\
& =\underset{\mu_{\bar{A}}}{\operatorname{Prob}}\left\{A^{\prime} \in \mathcal{F}_{n-1, m}^{\circ} \text { and } a_{n} \in T_{o}\left(\partial K_{A^{\prime}}, \varphi\right)\right\} \\
& =\int_{A^{\prime} \in \mathcal{F}_{n-1, m}^{\circ}} \operatorname{Prob}_{\mu_{\bar{a}}}\left\{a_{n} \in T_{o}\left(\partial K_{A^{\prime}}, \varphi\right)\right\} d \mu_{\bar{A}^{\prime}} .
\end{aligned}
$$

We fix $A^{\prime} \in \mathcal{F}_{n-1, m}^{\circ}$ and consider the properly convex set $K_{n}=K_{A^{\prime}}$ in $S^{m}$. The bound in Corollary 3.4 on the outer neighborhood of $\partial K_{n}$ yields

$$
\underset{\nu}{\operatorname{Prob}}\left\{a_{n} \in T_{o}\left(\partial K_{n}, \varphi\right)\right\}=\frac{\operatorname{vol}\left(T_{o}\left(\partial K_{n}, \varphi\right) \cap B\left(\bar{a}_{n}, \alpha\right)\right)}{\operatorname{vol} B\left(\bar{a}_{n}, \alpha\right)} \leq \frac{13 m}{2 \sigma} \sin \varphi,
$$

where $\nu$ denotes the uniform distribution on $B\left(\bar{a}_{n}, \alpha\right)$. The reader should note that $\varepsilon \leq 2 \sigma \delta_{c} /(13 m) \leq \sigma /(2 m)$ by assumption. (We win a factor of two by considering $B\left(\bar{a}_{n}, \alpha\right)$ instead of $B\left( \pm \bar{a}_{n}, \alpha\right)$.) Hence, using $\sin \varphi=$ $\varepsilon=(m+1) t^{-1}$, we conclude

$$
\underset{\nu}{\operatorname{Prob}}\left\{a_{n} \in T_{o}\left(\partial K_{n}, \varphi\right)\right\} \leq \frac{13 m}{2 \sigma} \sin \varphi=\frac{13 m(m+1)}{2 \sigma t} .
$$

We assume that $13 m(m+1)(2 \sigma t)^{-1} \leq \delta_{c}$. Hence we can apply Lemma 2.9, which yields

$$
\underset{\mu_{\bar{a}_{n}}}{\operatorname{Prob}}\left\{a_{n} \in T_{o}\left(\partial K_{n}, \varphi\right)\right\} \leq\left(\frac{13 m(m+1)}{2 \sigma t}\right)^{c}
$$


This bound holds for any $A^{\prime} \in \mathcal{F}_{n-1, m}^{\circ}$. We therefore obtain from (9)

$$
\begin{aligned}
& \underset{\mu_{\bar{A}}}{\operatorname{Prob}}\left\{A \in \mathcal{F}_{n, m}^{\circ} \text { and } a_{n} \in T_{o}\left(\partial K_{n}, \varphi\right)\right\} \\
& \leq\left(\frac{13 m(m+1)}{2 \sigma t}\right)^{c} \operatorname{Prob}_{\mu_{\bar{A}^{\prime}}}\left\{A^{\prime} \text { feasible }\right\} \leq\left(\frac{13 m(m+1)}{2 \sigma t}\right)^{c} .
\end{aligned}
$$

The same upper bound holds for any $K_{i}$. Altogether, we obtain

$$
\operatorname{Prob}_{\mu_{\bar{A}}}\left\{A \in \mathcal{F}_{n, m}^{\circ} \text { and } \mathscr{C}(A) \geq t\right\} \leq n\left(\frac{13 m(m+1)}{2 \sigma}\right)^{c} t^{-c},
$$

which proves Claim (F), since $\operatorname{Prob}\left\{A \in \Sigma_{n, m}\right\}=0$.

\subsection{Infeasible instances}

We start with a general observation. For $A=\left(a_{1}, \ldots, a_{n}\right) \in\left(S^{m}\right)^{n}$ and $1 \leq k \leq n$ we will write $A_{k}:=\left(a_{1}, \ldots, a_{k}\right)$ and $\bar{A}_{k}:=\left(\bar{a}_{1}, \ldots, \bar{a}_{k}\right)$.

Lemma 3.7 Let $A \in\left(S^{m}\right)^{n}, k<n$, such that $A_{k+1}$ be infeasible. Then

$$
\mathscr{C}\left(A_{k+1}\right) \geq \mathscr{C}(A)
$$

Proof. As $A_{k+1}$ is infeasible, $A$ must be infeasible as well. Let $A^{\prime}=$ $\left(a_{1}^{\prime}, \ldots, a_{n}^{\prime}\right)$ be feasible such that $d\left(A, A^{\prime}\right)=d\left(A, \Sigma_{n, m}\right) \leq \pi / 2$. Then $A_{k}^{\prime}=\left(a_{1}^{\prime}, \ldots, a_{k+1}^{\prime}\right)$ is feasible and $d\left(A_{k}, A_{k}^{\prime}\right) \leq d\left(A, A^{\prime}\right)$. Hence we have $d\left(A_{k+1}, \Sigma_{k+1, m}\right) \leq d\left(A, \Sigma_{n, m}\right)$ and

$$
\mathscr{C}\left(A_{k+1}\right)^{-1}=\sin d\left(A_{k+1}, \Sigma_{k+1, m}\right) \leq \sin d\left(A, \Sigma_{n, m}\right)=\mathscr{C}(A)^{-1},
$$

which was to be shown.

We also need the following probabilistic lemma.

Lemma 3.8 Let $U$ and $V$ be random variables taking positive values and $x_{U} \geq \alpha>0, x_{V} \geq \beta>0$, and $c>0$. We assume

$$
\begin{aligned}
\operatorname{Prob}\{U \geq x\} & \leq \alpha \cdot x^{-c} \quad \text { for } x \geq x_{U} \\
\operatorname{Prob}\{V \geq x \mid U\} & \leq \beta \cdot x^{-c} \quad \text { for } x \geq x_{V} .
\end{aligned}
$$

Then we have

$$
\operatorname{Prob}\{U V \geq x\} \leq c \alpha \beta x^{-c} \ln \max \left\{\frac{x}{x_{U} x_{V}}, 1\right\}+\min \left\{\alpha x_{V}^{c}, \beta x_{U}^{c}\right\} x^{-c} .
$$


Proof. [23, Lemma C.1] with the functions $f, g$ defined as

$$
f(x)=\left\{\begin{array}{ll}
1 & \text { if } x<x_{U} \\
\alpha \cdot x^{-c} & \text { if } x \geq x_{U}
\end{array} \quad g(x)= \begin{cases}1 & \text { if } x<x_{V} \\
\beta \cdot x^{-c} & \text { if } x \geq x_{V}\end{cases}\right.
$$

yields

$$
\operatorname{Prob}\{U V \geq x\} \leq \int_{0}^{\infty} f\left(\frac{x}{s}\right)\left(-g^{\prime}(s)\right) d s .
$$

If $x \geq x_{U} x_{V}$ we estimate this by

$$
\begin{aligned}
\operatorname{Prob}\{U V \geq x\} & \leq \int_{x_{V}}^{x / x_{U}} \alpha x^{-c} s^{c} c \beta s^{-c-1} d s+\int_{x / x_{U}}^{\infty} c \beta s^{-c-1} d s \\
& =c \alpha \beta x^{-c} \ln \left(\frac{x}{x_{U} x_{V}}\right)+\beta x_{U}^{c} x^{-c} .
\end{aligned}
$$

If $x<x_{U} x_{V}$ one argues similarly.

Finally note that (11) implies $\operatorname{Prob}\{U V \geq x\} \leq \int_{0}^{\infty} g\left(\frac{x}{s}\right)\left(-f^{\prime}(s)\right) d s$, using integration by parts. Estimating this as before, with the roles of $f$ and $g$ exchanged, completes the proof.

We provide now the proof of the part of Theorem 3.1 dealing with infeasible instances, i.e., of the claimed bound (I). Fix $\bar{A} \in S^{m}, 0<\alpha \leq \pi / 2$, $\sigma=\sin \alpha$, and $t \geq 1$. Assume $A=\left(a_{1}, \ldots, a_{n}\right)$ to be chosen at random according to $\mu_{\bar{A}}$. Then $A_{m+1}$ is always feasible. Hence, if $A=A_{n}$ is infeasible, then there exists a smallest index $k>m$ such that $A_{k}$ is feasible and $A_{k+1}$ is infeasible. If we denote by $\mathcal{E}_{k}$ the event

$$
A_{k} \text { feasible and } A_{k+1} \text { infeasible and } \mathscr{C}\left(A_{k+1}\right) \geq t,
$$

and take into account Lemma 3.7, we obtain

$$
\operatorname{Prob}\left\{A \in \mathcal{I}_{n, m} \text { and } \mathscr{C}(A) \geq t\right\} \leq \sum_{k=m+1}^{n-1} \operatorname{Prob} \mathcal{E}_{k} .
$$

For bounding the probability of $\mathcal{E}_{k}$, a change of notation is convenient. We fix $k$ and write from now on

$$
A:=\left(a_{1}, \ldots, a_{k}\right), \quad K_{A}:=-\operatorname{sconv}\left\{a_{1}, \ldots, a_{k}\right\}, \quad b:=a_{k+1},
$$

and similarly $\bar{A}:=\left(\bar{a}_{1}, \ldots, \bar{a}_{k}\right), \bar{b}:=\bar{a}_{k+1}$. We note that $A$ and $b$ are chosen independently and at random according to $\mu_{\bar{A}}$ and $\mu_{\bar{b}}$, respectively. 
Proposition 3.6 implies that

$$
\underset{\mu_{\bar{A}}}{\operatorname{Prob}} \mathcal{E}_{k} \leq \underset{\mu_{\bar{A}}}{\operatorname{Prob}}\left\{A \in \mathcal{F}_{k, m} \text { and } b \in K_{A} \text { and } \frac{\mathscr{C}(A)}{\sin d\left(b, K_{A}\right)} \geq \frac{t}{10}\right\} .
$$

The first part of Theorem 3.1 tells us that

$$
\underset{\mu_{\bar{A}}}{\operatorname{Prob}}\left\{A \in \mathcal{F}_{k, m} \text { and } \mathscr{C}(A) \geq x\right\} \leq k\left(\frac{13 m(m+1)}{2 \sigma}\right)^{c} x^{-c}
$$

provided $x \geq x_{U}:=13 m(m+1) /\left(2 \sigma \delta_{c}\right)$. For a fixed strictly feasible $A$, the set $K_{A}$ is properly convex in $S^{m}$. The bound in Corollary 3.4 on the inner neighborhood of $\partial K_{A}$ yields for any $A \in \mathcal{F}_{k, m}^{\circ}$

$$
\underset{\nu}{\operatorname{Prob}}\left\{b \in K_{A} \text { and } \frac{1}{\sin d\left(b, \partial K_{A}\right)} \geq x \mid A\right\} \leq \frac{13 m}{2 \sigma} \frac{1}{x},
$$

provided $x \geq 2 m / \sigma$ (again $\nu$ denotes the uniform distribution on $B(\bar{b}, \alpha)$ ). Applying Lemma 2.9 yields

$$
\operatorname{Prob}_{\mu_{\bar{b}}}\left\{b \in K_{A} \text { and } \frac{1}{\sin d\left(b, \partial K_{A}\right)} \geq x \mid A\right\} \leq\left(\frac{13 m}{2 \sigma}\right)^{c} x^{-c},
$$

provided $x \geq x_{V}:=13 \mathrm{~m} /\left(2 \sigma \delta_{c}\right)$.

Let $\mathbf{1}_{M}$ denote the indicator function of a set $M$. We combine the above two probability estimates in (14) and (16) with Lemma 3.8, setting

$$
U(A):=\mathbf{1}_{\mathcal{F}_{k, m}}(A) \mathscr{C}(A), \quad V(A, b):=\mathbf{1}_{K_{A}}(b) \frac{1}{\sin d\left(b, \partial K_{A}\right)} .
$$

Note that

$$
\operatorname{Prob} \mathcal{E}_{k} \leq \operatorname{Prob}\{U(A) \cdot V(A, b) \geq t / 10\} .
$$

We have for $t \geq 1$ and $x=t / 10$

$$
\max \left\{\frac{x}{x_{U} x_{V}}, 1\right\}=\max \left\{\frac{4 \sigma^{2} \delta_{c}^{2} t}{1690 m^{2}(m+1)}, 1\right\} \leq t .
$$

Let $\alpha:=k\left(\frac{13 m(m+1)}{2 \sigma}\right)^{c}$ and $\beta:=\left(\frac{13 m}{2 \sigma}\right)^{c}$. Note that $\alpha \beta=k \cdot\left(\frac{169 m^{2}(m+1)}{4 \sigma^{2}}\right)^{c}$ and $\min \left\{\alpha x_{V}^{c}, \beta x_{U}^{c}\right\}=\left(\frac{169 m^{2}(m+1)}{4 \sigma^{2} \delta_{c}}\right)^{c}$. Lemma 3.8 implies that

$$
\operatorname{Prob} \mathcal{E}_{k} \leq c k\left(\frac{1690 m^{2}(m+1)}{4 \sigma^{2}}\right)^{c} t^{-c} \ln t+\left(\frac{1690 m^{2}(m+1)}{4 \sigma^{2} \delta_{c}}\right)^{c} t^{-c} .
$$

Plugging in this bound into (13) finishes the proof of Theorem 3.1. 


\subsection{Proof of Theorem 1.2}

We will now give the proof of Theorem 1.2 by deriving estimates of the expectation of $\ln \mathscr{C}$ from the tail bounds given in Theorem 3.1. Let $A \in\left(S^{m}\right)^{n}$ be chosen at random according to an adversarial distribution $\mu_{\bar{A}}$. Combining $(\mathrm{F})$ and $(\mathrm{I})$ from Theorem 3.1 we have for $t \geq 13 m(m+1)\left(2 \sigma \delta_{c}\right)^{-1}$,

$$
\begin{gathered}
\operatorname{Prob}\{\mathscr{C}(A) \geq t\}=\operatorname{Prob}\left\{A \in \mathcal{F}_{n, m} \text { and } \mathscr{C}(A) \geq t\right\} \\
\quad+\operatorname{Prob}\left\{A \in \mathcal{I}_{n, m} \text { and } \mathscr{C}(A) \geq t\right\} \\
\leq n\left(\frac{13 m(m+1)}{2 \sigma}\right)^{c} t^{-c}+n\left(\frac{1690 m^{2}(m+1)}{4 \sigma^{2} \delta_{c}}\right)^{c} t^{-c} \\
\quad+c n^{2}\left(\frac{1690 m^{2}(m+1)}{4 \sigma^{2}}\right)^{c} t^{-c} \ln t .
\end{gathered}
$$

Defining $D_{1}:=n\left(\frac{13 m(m+1)}{2 \sigma}\right)^{c}, D_{2}:=D_{1} \cdot\left(\frac{130 m}{2 \sigma \delta_{c}}\right)^{c}, D_{3}:=c n D_{1} \cdot\left(\frac{130 m}{2 \sigma}\right)^{c}$ we can write shortly

$$
\operatorname{Prob}\{\mathscr{C}(A) \geq t\} \leq D_{1} t^{-c}+D_{2} t^{-c}+D_{3} t^{-c} \ln t
$$

Note that $D_{2} \geq D_{1}$ and $D_{3} \geq D_{1}$. It is convenient to use tail estimates of $\ln \mathscr{C}$ instead of $\mathscr{C}$, so we reformulate

$$
\operatorname{Prob}\{\ln \mathscr{C}(A) \geq s\} \leq D_{1} e^{-c s}+D_{2} e^{-c s}+D_{3} e^{-c s} s
$$

which holds for $s \geq \ln \left(\frac{13 m(m+1)}{2 \sigma \delta_{c}}\right)$. We define $s_{i}:=\ln D_{i}^{1 / c}+\ln \delta_{c}^{-1}$, i.e. $\delta_{c}=$ $D_{i}^{1 / c} \cdot e^{-s_{i}}$, for $i=1,2,3$. Note that $s_{2}=s_{1}+\ln \left(\frac{130 m}{2 \sigma \delta_{c}}\right) \geq s_{1}, s_{3}=$ $s_{1}+c^{-1} \ln (c n)+\ln \left(\frac{130 m}{2 \sigma}\right) \geq s_{1}$, and

$$
s_{1}=\frac{1}{c} \ln n+\ln \left(\frac{13 m(m+1)}{2 \sigma}\right)+\ln \delta_{c}^{-1} \geq \ln \left(\frac{13 m(m+1)}{2 \sigma \delta_{c}}\right) .
$$

The definition of $\delta_{c}$ in Lemma 2.9 and the inequality

$$
\left(1-\left(\frac{2}{\pi m}\right)^{\frac{1}{m}}\right)^{-1 / 2} \leq \sqrt{\frac{2 m}{\ln (\pi m / 2)}}
$$

which is shown by a small computation, lead to the following estimate of $s_{1}$

$$
\begin{aligned}
s_{1} & \leq \ln D_{1}^{1 / c}+\ln \frac{\pi m}{2}+\frac{1}{2 c} \ln \left(\frac{2 H^{2} m}{\ln (\pi m / 2)}\right) \\
& =c^{-1} \ln n+\ln \left(\frac{13 m(m+1)}{2 \sigma}\right)+\ln \frac{\pi m}{2}+\frac{1}{2 c} \ln \left(\frac{2 H^{2} m}{\ln (\pi m / 2)}\right) .
\end{aligned}
$$


We distinguish the cases $s_{3} \geq s_{2}$ and $s_{3}<s_{2}$. For $s_{3} \geq s_{2}$ we get with (18)

$$
\begin{aligned}
\mathbb{E}(\ln \mathscr{C}) & =\int_{0}^{\infty} \operatorname{Prob}\{\ln \mathscr{C}(A) \geq s\} d s \\
\leq & \int_{0}^{s_{3}} 1 d s+\int_{s_{3}}^{\infty}\left(D_{1} e^{-c s}+D_{2} e^{-c s}+D_{3} e^{-c s} s\right) d s \\
\leq & s_{3}+\int_{s_{1}}^{\infty} D_{1} e^{-c s} d s+\int_{s_{2}}^{\infty} D_{2} e^{-c s} d s+\int_{s_{3}}^{\infty} D_{3} e^{-c s} s d s \\
= & s_{3}+c^{-1} \delta_{c}^{c}+c^{-1} \delta_{c}^{c}+c^{-2}\left(1+c s_{3}\right) \delta_{c}^{c} \\
\leq & \left(1+c^{-1}\right) s_{3}+2 c^{-1}+c^{-2} \\
= & \left(1+c^{-1}\right) s_{1}+\left(1+c^{-1}\right) c^{-1} \ln (c n)+\left(1+c^{-1}\right) \ln \left(\frac{130 m}{2 \sigma}\right) \\
& \quad+2 c^{-1}+c^{-2} .
\end{aligned}
$$

With the estimate of $s_{1}$ in (20) a small computation yields

$$
\begin{aligned}
\mathbb{E}(\ln \mathscr{C}) \leq(1+ & \left.c^{-1}\right)\left(2 c^{-1} \ln n+3 \ln m+\ln (m+1)+2 \ln \frac{1}{\sigma}\right. \\
& \left.+(2 c)^{-1} \ln \left(\frac{2 H^{2} m}{\ln (\pi m / 2)}\right)+c^{-1}+c^{-1} \ln c+6.5\right)+c^{-1} .
\end{aligned}
$$

This yields $\mathbb{E}(\ln \mathscr{C})=\mathcal{O}\left(c^{-2} \ln \left(\frac{n H}{\sigma}\right)\right)$. In the case $s_{3}<s_{2}$ a similar argument holds. This shows the first statement of Theorem 1.2. Finally, tracing the constants in the case $\beta=0$, yields the asserted explicit bound.

\subsection{Average-case analysis}

We show here that for the uniform distribution on $S^{m}$, the probability tail estimates in Theorem 3.1 on $\mathscr{C}(A)$ can be significantly improved by essentially the same method.

Proposition 3.9 For $A \in\left(S^{m}\right)^{n}$ chosen uniformly at random we have

$$
\operatorname{Prob}\{\mathscr{C}(A) \geq t\}=\mathcal{O}\left((m+1)^{5} \frac{1}{t} \ln t\right) .
$$

Moreover, $\mathbb{E}(\ln \mathscr{C}(A))=\mathcal{O}(\ln m)$ as stated in Corollary 1.3.

Proof (Sketch). A result due to Wendel [31] states that for $k>m$

$$
p(k, m):=\frac{\operatorname{vol}_{k, m}}{\left(\operatorname{vol} S^{m}\right)^{k}}=\frac{1}{2^{k-1}} \sum_{i=0}^{m}\left(\begin{array}{c}
k-1 \\
i
\end{array}\right) .
$$


We refer to the proof in $\S 3.3$ for the uniform distribution on $S^{m}$ instead of $\mu_{\bar{A}}$ (think of $\sigma=1$ ). We write $k$ instead of $n$. We do not need to use Lemma 2.9. Moreover we do not bound $p(k, m)=\operatorname{Prob}\left\{A \in \mathcal{F}_{k, m}\right\}$ by 1 as in Equation (10). Taking account of this, the proof in $\S 3.3$ shows that

$$
\operatorname{Prob}\left\{A \in \mathcal{F}_{k, m} \text { and } \mathscr{C}(A) \geq x\right\} \leq k \cdot \frac{13 m(m+1)}{2} p(k, m) \frac{1}{x},
$$

provided $x \geq x_{U}:=2 m(m+1)$.

We proceed now as in $\S 3.4$, using the same notation. For a fixed strictly feasible $A=\left(a_{1}, \ldots, a_{k}\right)$ we have by $(15)$

$$
\operatorname{Prob}\left\{b \in K_{A} \text { and } \frac{1}{\sin d\left(b, \partial K_{A}\right)} \geq x \mid A\right\} \leq \frac{13 m}{2} \frac{1}{x}
$$

provided $x \geq x_{V}:=2 m$. Recall the definition of the event $\mathcal{E}_{k}$ from (12). Similarly as for (17) we conclude with the help of Lemma 3.8 that

$$
\operatorname{Prob} \mathcal{E}_{k} \leq C m^{3} k p(k, m) \frac{1}{t} \ln t \quad \text { for } t \geq e,
$$

where $C$ stands for a universal constant. Using Lemma 3.10 stated below we get

$$
\sum_{k=m+1}^{n-1} k p(k, m) \leq \sum_{k=m+1}^{4 m} k p(k, m)+\sum_{k=4 m+1}^{\infty} k p(k, m)=\mathcal{O}\left(m^{2}\right) .
$$

Hence, by Equation (13),

$$
\operatorname{Prob}\left\{A \in \mathcal{I}_{n, m} \text { and } \mathscr{C}(A) \geq t\right\} \leq \sum_{k=m+1}^{n-1} \operatorname{Prob} \mathcal{E}_{k} \leq C^{\prime} m^{5} \frac{1}{t} \ln t
$$

for some constant $C^{\prime}$. It is obvious that $\operatorname{Prob}\left\{A \in \mathcal{F}_{n, m}\right.$ and $\left.\mathscr{C}(A) \geq t\right\}$ can also be bounded this way. Finally, the claimed bound on the expectation of $\ln \mathscr{C}(A)$ follows immediately with the help of [6, Prop. 2.4].

Lemma 3.10 We have $\sum_{k=4 m+1}^{\infty} k p(k, m)=o(1)$ for $m \rightarrow \infty$.

Proof. Let $k>4 m$. Wendel's result (21) implies

$$
k p(k, m) \leq k \frac{(m+1)}{2^{k-1}}\left(\begin{array}{c}
k-1 \\
m
\end{array}\right) \leq \frac{2(m+1)}{m !} \frac{k^{m+1}}{2^{k}} .
$$


We have $k^{m+1} 2^{-k} \leq 2^{-k / 2}$ for $k \geq C m \log m$, and sufficiently large $m$, where $C>0$ is a suitable universal constant. Therefore, we get

$$
\sum_{k \geq C m \log m} k p(k, m) \leq \frac{2(m+1)}{m !} \sum_{k=0}^{\infty} \frac{1}{2^{k / 2}}=o(1)(m \rightarrow \infty) .
$$

The function $x \mapsto x^{m+1} 2^{-x}$ is monotonically decreasing for $x \geq(m+1) / \ln 2$. Hence, as $k>4 m$, and using $m ! \geq(m / e)^{m}$ we get

$$
\frac{1}{m !} \frac{k^{m+1}}{2^{k}} \leq \frac{1}{m !} \frac{(4 m)^{m+1}}{2^{4 m}} \leq 4 m\left(\frac{e}{4}\right)^{m}
$$

Since $e / 4<1$, we conclude

$$
\sum_{k=4 m+1}^{C m \log m} k p(k, m) \leq 8 m(m+1)\left(\frac{e}{4}\right)^{m} C m \log m=o(1)(m \rightarrow \infty),
$$

which completes the proof.

\section{Some spherical convex geometry}

The goal of this section is to provide the proof of Theorem 3.3, following the lines of [7, Theorem 1.2]. We proceed in several steps.

\subsection{Integrals of curvature and Weyl's tube formula}

For the following material from differential geometry we refer to [29]. A good reference for the differential geometry of convex sets is [3].

Let $V$ be a smooth hypersurface in $S^{m}$ with unit normal vector field $\nu: V \rightarrow S^{m}$. The principal curvatures of $V$ at $x \in V$ are defined as the eigenvalues $\kappa_{1}(x), \ldots, \kappa_{m-1}(x)$ of the Weingarten map $-D \nu(x): T_{x} V \rightarrow$ $T_{x} V$. The $i$ th curvature $\mathscr{K}_{V, i}(x)$ of $V$ at $x$ is the $i$ th symmetric polynomial in the principal curvatures:

$$
\mathscr{K}_{V, i}(x):=\sum_{|I|=i} \prod_{j \in I} \kappa_{j}(x) \quad(0 \leq i<m) .
$$

Interesting special cases are $\mathscr{K}_{V, 0}(x)=1$ and

$$
\mathscr{K}_{V, m-1}(x)=\kappa_{1}(x) \cdots \kappa_{m-1}(x)=\operatorname{det}(-D \nu(x)),
$$


which is called the Gaussian curvature of $V$ at $x$. Let $U$ be an open subset of $V$. In [7] the integral $\mu_{i}(U)$ of $i$ th curvature and the integral $\left|\mu_{i}\right|(U)$ of $i$ th absolute curvature were defined as

$$
\mu_{i}(U):=\int_{U} \mathscr{K}_{V, i} d V, \quad\left|\mu_{i}\right|(U):=\int_{U}\left|\mathscr{K}_{V, i}\right| d V .
$$

Two special cases deserve special mention: $\mu_{0}(U)=$ vol $U$ equals the $(m-1)$ dimensional volume of $U$. Moreover, $\mu_{m-1}(V)$ is the integral of the Gaussian curvature of $V$.

By a smooth convex body $K$ in $S^{m}$ we will understand a convex body such that its boundary $\partial K$ is a smooth hypersurface in $S^{m}$ (of type $C^{\infty}$ ) and its Gaussian curvature does not vanish in any point of $\partial K$.

Let $K$ be a smooth convex body in $S^{m}$ with boundary $V:=\partial K$. We denote by $\nu: V \rightarrow S^{m}$ the unit normal vector field of the hypersurface $V$ that points inwards of $K$. Here all the principal curvatures $\kappa_{j}(x)$ are nonnegative, cf. [3]. Hence the $i$ th curvatures are nonnegative as well and therefore we have $\mu_{i}(U)=\left|\mu_{i}\right|(U)$ for any open subset $U$ of $V$.

For $0<\varphi \leq \pi / 2$ we define the $\varphi$-tube $T^{\perp}(U, \varphi)$ around $U$ as

$$
\begin{aligned}
T^{\perp}(U, \varphi):=\left\{x \in S^{m} \mid\right. & \exists y \in U \text { such that } d(x, y)<\varphi \text { and } \\
& {[x, y] \text { intersects } U \text { orthogonally at } y\} . }
\end{aligned}
$$

The outer $\varphi$-tube $T_{o}^{\perp}(U, \varphi)$ and inner $\varphi$-tube $T_{i}^{\perp}(U, \varphi)$ of $U$ are defined as

$$
T_{o}^{\perp}(U, \varphi):=T^{\perp}(U, \varphi) \backslash K \text { and } T_{i}^{\perp}(U, \varphi):=T^{\perp}(U, \varphi) \cap K .
$$

In an important paper, Weyl [32] derived a formula for the volume of tubes around compact submanifolds of euclidean spaces or spheres. His result can be seen as extension of Steiner's formula on the volume of "parallel convex sets" in euclidean space, see also Allendoerfer [1]. Weyl's formula only holds for a sufficiently small radius. In [7, Prop. 3.1], it was observed that when replacing integrals of curvature by absolute integrals of curvature, one obtains an upper bound on the volume of tubes holding for any radius. As the above two notions of curvature coincide for boundaries of convex sets, we get the following result. (An inspection of the proof of [7, Prop. 3.1] reveals that separate bounds on the inner and outer tube hold.)

Proposition 4.1 Let $K$ be a smooth convex body in $S^{m}$ and $U$ be an open subset of $\partial K$. Then we have for all $0<\varphi \leq \pi / 2$

$$
\max \left\{\operatorname{vol} T_{o}^{\perp}(U, \varphi), \operatorname{vol} T_{i}^{\perp}(U, \varphi)\right\} \leq \sum_{i=0}^{m-1} J_{m, i+1}(\varphi) \mu_{i}(U),
$$


where $J_{m, i+1}$ denotes the function defined in (4). Moreover, this upper bound is sharp for sufficiently small $\varphi$, cf. [32].

\subsection{Some integral geometry}

We will need a special case of the principal kinematic formula of integral geometry for spheres. We denote by $G$ the orthogonal group $O(m+1)$, that operates on $S^{m}$ in the natural way, and denote by $d G$ its volume element normalized such that the volume of $G$ equals one. The following result is Theorem 2.7. in [7]. (For related information see [19] and [16].)

Theorem 4.2 Let $U$ be an open subset of a compact oriented smooth hypersurface $M$ of $S^{m}$ and $0 \leq i<m-1$. Then we have

$$
\mu_{i}(U)=\mathcal{C}(m, i) \int_{g \in G} \mu_{i}\left(g U \cap S^{i+1}\right) d G(g),
$$

where $\mathcal{C}(m, i)=(m-i-1)\left(\begin{array}{c}m-1 \\ i\end{array}\right) \frac{\mathcal{O}_{m-1} \mathcal{O}_{m}}{\mathcal{O}_{i} \mathcal{O}_{i+1} \mathcal{O}_{m-i-2}}$.

The special case $i=0$ yields an effective tool for estimating volumes, usually referred to as Poincaré's formula:

$$
\operatorname{vol}_{m-1} U=\frac{\mathcal{O}_{m-1}}{2} \int_{g \in G} \#\left(U \cap g S^{1}\right) d G(g),
$$

where \# $\left(U \cap g S^{1}\right)$ denotes the number of elements in $U \cap g S^{1}$ (note that this is a finite set for almost all $g \in G$ ). Here is an application of (23). Clearly, the given bound is sharp (consider spherical caps with radius almost $\pi / 2$ ).

Corollary 4.3 Any smooth convex body $K$ in $S^{m}$ satisfies vol $\partial K \leq \mathcal{O}_{m-1}$.

Proof. Almost surely, the intersection $\partial K \cap g S^{1}$ is finite. Then it consists of at most two points by convexity.

\subsection{Integrals of curvature for boundaries of convex sets}

In this section we assume that $K$ is a smooth convex body in $S^{m}$ and $\nu$ is the unit normal vector field on $\partial K$ pointing inwards of $K$. This means that for $x \in V$, the unit vector $\nu(x)$ is uniquely characterized by the conditions $\langle v, x\rangle=0$ and $\langle v, y\rangle \geq 0$ for all $y \in K$.

Lemma 4.4 We have $-\nu(\partial K)=\partial \breve{K}$. 
Proof. The characterization of $\nu(x)$ implies that $-\nu(x) \in \partial \breve{K}$ for $x \in \partial K$. For the other inclusion, let $v$ be a unit vector satisfying $-v \in \partial \breve{K}$. Then $\langle v, y\rangle \geq 0$ for all $y \in K$. Moreover, there exists $x \in K$ such that $\langle v, x\rangle=0$. This implies $x \in \partial K$. It follows that $v=\nu(x)$.

The following bound is crucial for all what follows. Again, considering spherical caps with radius almost $\pi / 2$, shows the optimality of the bound.

Proposition 4.5 The integral of Gaussian curvature of $\partial K$ is bounded as $\mu_{m-1}(\partial K) \leq \mathcal{O}_{m-1}$.

Proof. Put $V:=\partial K$. By Lemma 4.4, $\nu: V \rightarrow \partial \breve{K}$ is surjective. By (22) we have $\mathscr{K}_{V, m-1}(x)=\operatorname{det}(-D \nu(x))$ for $x \in V$. Since we assume that the Gaussian curvature does not vanish, the map $\nu$ has no singular values.

We claim that $\nu$ is injective. Otherwise, we had $\nu(x)=\nu(y)$ for distinct $x, y \in V$. Since $\langle\nu(x), x\rangle=0$ and $\langle\nu(y), y\rangle=0$ we had $\langle\nu(x), z\rangle=0$ for all $z \in[x, y]$. Hence $\nu$ would be constant along this segment and therefore $x$ would be a critical point, contradicting our asumption.

We conclude that $-\nu: V \rightarrow \nu(V)$ is a diffeomorphism onto the smooth hypersurface $\partial \breve{K}$. The transformation theorem yields

$$
\mu_{m-1}(V)=\int_{V} \mathscr{K}_{V, m-1} d V=\int_{V} \operatorname{det}(-D \nu) d V=\operatorname{vol} \partial \breve{K} .
$$

Corollary 4.3 implies now the assertion.

Lemma 4.6 For $a \in S^{m}, 0<\alpha \leq \pi / 2, \sigma=\sin \alpha$, and $0 \leq i<m$ we have

$$
\mu_{i}(\partial K \cap B(a, \alpha)) \leq\left(\begin{array}{c}
m-1 \\
i
\end{array}\right) \mathcal{O}_{m-1} \sigma^{m-i-1}
$$

Proof. This is similar, but somewhat simpler than the proof of [7, Prop. 3.2]. The case $i=m-1$ is already established by Proposition 4.5. Hence we assume $i<m-1$. Let $g \in G=O(m+1)$ be such that $V:=\partial K$ intersects $g S^{i+1}$ transversally with nonempty intersection. We apply Proposition 4.5 to the convex body $K \cap g S^{i+1}$ in the sphere $g S^{i+1}$, which has the smooth boundary $V \cap g S^{i+1}$. Hence $\mu_{i}\left(V \cap g S^{i+1}\right) \leq \mathcal{O}_{i}$. The kinematic formula of Theorem 4.2 applied to the open subset $U:=V \cap \operatorname{int}(B(a, \alpha))$ of $V$ yields

$$
\begin{aligned}
\mu_{i}(U) & =\mathcal{C}(m, i) \int_{g \in G} \mu_{i}\left(g U \cap S^{i+1}\right) d G(g) \\
& \leq \mathcal{C}(m, i) \mathcal{O}_{i} \underset{g \in G}{\operatorname{Prob}}\left\{g U \cap S^{i+1} \neq \emptyset\right\} .
\end{aligned}
$$


Using $g U \subseteq B(g a, \alpha)$, this probability may be estimated as follows

$$
\begin{aligned}
& \underset{g \in G}{\operatorname{Prob}}\left\{g U \cap S^{i+1} \neq \emptyset\right\} \leq \underset{g \in G}{\operatorname{Prob}}\left\{B(g a, \alpha) \cap S^{i+1} \neq \emptyset\right\} \\
& =\underset{a^{\prime} \in S^{m}}{\operatorname{Prob}}\left\{B\left(a^{\prime}, \alpha\right) \cap S^{i+1} \neq \emptyset\right\}=\mathcal{O}_{m}^{-1} \operatorname{vol} T\left(S^{i+1}, \alpha\right) .
\end{aligned}
$$

Lemma 2.1 in [7] implies vol $T\left(S^{i+1}, \alpha\right)=\mathcal{O}_{i+1} \mathcal{O}_{m-i-2} J_{m, m-i-1}(\alpha)$. Moroever, Lemma 2.2 in [7] says that

$$
J_{m, k}(\alpha) \leq \frac{\sigma^{k}}{k} \quad \text { for } 1 \leq k<m .
$$

By combining these estimates and plugging in the formula for $\mathcal{C}(m, i)$ from Theorem 4.2, the resulting expression considerably simplifies and we get $\mu_{i}(U) \leq\left(\begin{array}{c}m-1 \\ i\end{array}\right) \mathcal{O}_{m-1} \sigma^{m-i-1}$ as claimed.

\subsection{Proof of Theorem 3.3}

We can finally provide the proof of Theorem 3.3. We assume first that $K$ is a smooth convex body in $S^{m}$. Let $a \in S^{m}, 0<\alpha, \varphi \leq \pi / 2$, put $\sigma=\sin \alpha$, $\varepsilon=\sin \varphi$, and let $U=\partial K \cap B(a, \alpha)$. By combining Proposition 4.1 with Lemma 4.6 we get

$$
\operatorname{vol} T_{o}^{\perp}(U, \varphi) \leq \sum_{i=0}^{m-1}\left(\begin{array}{c}
m-1 \\
i
\end{array}\right) \mathcal{O}_{m-1} \sigma^{m-i-1} J_{m, i+1}(\varphi) .
$$

Using the estimate (24) we obtain after a short calculation (put $k=i+1$, use $\left(\begin{array}{c}m-1 \\ k-1\end{array}\right)=\frac{k}{m}\left(\begin{array}{c}m \\ k\end{array}\right)$ and consider separately the term for $k=m$ )

$$
\operatorname{vol} T_{o}^{\perp}(\partial K \cap B(a, \alpha), \varphi) \leq \frac{\mathcal{O}_{m-1}}{m} \sum_{k=1}^{m-1}\left(\begin{array}{c}
m \\
k
\end{array}\right) \varepsilon^{k} \sigma^{m-k}+\frac{1}{2} \mathcal{O}_{m} \varepsilon^{m} .
$$

The same upper bound holds for the volume of $T_{i}^{\perp}(\partial K \cap B(a, \alpha), \varphi)$.

We claim that

$$
T_{o}(\partial K, \varphi) \cap B( \pm a, \alpha) \subseteq T_{o}^{\perp}(\partial K \cap B( \pm a, \beta), \varphi)
$$

where $\beta=\arcsin \min \{1, \sigma+\varepsilon\}$. Indeed, suppose $x \in T_{o}(\partial K, \varphi) \cap B( \pm a, \alpha)$ and let $y \in \partial K$ be a closest point to $x$. Then $d(x, y) \leq \varphi$ and $[x, y]$ intersects $\partial K$ orthogonally (as $\partial K$ is smooth without boundary). The triangle inequality for projective distance (cf. §2.2) implies that $\sin d(a, y)<$ 
$\sin d(a, x)+\sin d(x, y) \leq \sigma+\varepsilon$. Hence $\sin d(a, y) \leq \sin \beta$ and therefore $y \in B( \pm a, \beta)$ which shows the claim.

By combining (26) with (25) we get

$$
\operatorname{vol}\left(T_{o}(\partial K, \varphi) \cap B( \pm a, \alpha)\right) \leq \frac{2 \mathcal{O}_{m-1}}{m} \sum_{k=1}^{m-1}\left(\begin{array}{c}
m \\
k
\end{array}\right) \varepsilon^{k}(\sigma+\varepsilon)^{m-k}+\mathcal{O}_{m} \varepsilon^{m}
$$

We have $\operatorname{vol} B( \pm a, \alpha) \geq 2 \mathcal{O}_{m-1} \frac{\sigma^{m}}{m}$, cf. [7, Lemmas 2.1-2.2]. Using this, we obtain

$\frac{\operatorname{vol}\left(T_{o}(\partial K, \varphi) \cap B( \pm a, \alpha)\right)}{\operatorname{vol} B( \pm a, \alpha)} \leq \sum_{k=1}^{m-1}\left(\begin{array}{c}m \\ k\end{array}\right)\left(1+\frac{\varepsilon}{\sigma}\right)^{m-k}\left(\frac{\varepsilon}{\sigma}\right)^{k}+\frac{m \mathcal{O}_{m}}{2 \mathcal{O}_{m-1}}\left(\frac{\varepsilon}{\sigma}\right)^{m}$.

This shows the assertion of Theorem 3.3 for the outer neighborhood in the case where $K$ is a smooth convex body. The bound for the inner neighborhood is shown similarly.

The general case where $K$ is any properly convex set in $S^{m}$ will follow by a pertubation argument. We define the Hausdorff distance $d\left(K, K^{\prime}\right)$ of two convex sets $K$ and $K^{\prime}$ in $S^{m}$ as the infimum of the real numbers $\delta \geq 0$ satisfying $K \subseteq T\left(K^{\prime}, \delta\right)$ and $K^{\prime} \subseteq T(K, \delta)$. This defines a metric and allows to speak about the convergence of convex sets. (For compact convex sets in euclidean space the Hausdorff distance is a well known notion.)

Lemma 4.7 Any properly convex set $K$ in $S^{m}$ is the limit of a sequence of smooth convex bodies.

Proof. The euclidean version of the claim is a well known result due to Minkowski, see [3, §6] (or [24]) for more information.

A properly convex set $K \subset S^{m}$ is contained in an open halfspace. For fixed $p \in S^{m}$ consider now the open halfsphere $S_{+}^{m}:=\left\{x \in S^{m}|\langle x, p\rangle\rangle\right.$ $0\}$ with center $p$ and the affine space $E:=\left\{x \in \mathbb{R}^{m+1} \mid\langle x, p\rangle=1\right\}$. The "perspective map" $\pi: S_{+}^{m} \rightarrow E, x \mapsto\langle p, x\rangle^{-1} x$ maps an intersection of a linear space with $S^{m}$ to an affine linear subspace of $E$ and vice versa. Moreover, $\pi$ maps convex sets to convex sets and vice versa. Moreover, one sees that $\pi$ induces a homeomorphism between the set of convex subsets of $S_{+}^{m}$ and the set of compact convex subsets of $E$. It is easily checked that if $\tilde{K} \subset E$ smooth compact convex has nonvanishing Gaussian curvature on the boundary, then this also holds for $\pi(\tilde{K})$. The assertion follows from the euclidean version of our claim. 
To finish the proof of Theorem 3.3 let now $K \subset S^{m}$ be a properly convex set and $\delta>0$. By Lemma 4.7 there exists a smooth convex body $K^{\prime}$ such that $K$ and $K^{\prime}$ have Hausdorff distance at most $\delta$, which means that $K \subseteq T\left(K^{\prime}, \delta\right)$ and $K^{\prime} \subseteq T(K, \delta)$. This implies $K^{\prime} \backslash K \subseteq T(\partial K, \delta)$ and

$$
T_{o}(\partial K, \varphi) \subseteq T_{o}\left(\partial K^{\prime}, \varphi+\delta\right) \cup\left(K^{\prime} \backslash K\right) .
$$

By applying $(27)$ to $T_{o}\left(\partial K^{\prime}, \varphi+\delta\right)$, letting $\delta \rightarrow 0$, and noting that vol $T(\partial K, \delta)$ goes to zero, the desired assertion follows. For the inner neighborhood one argues similarly.

\section{References}

[1] C.B. Allendoerfer. Steiner's formulae on a general $S^{n+1}$. Bull. Amer. Math. Soc., 54:128-135, 1948.

[2] K. Ball. The reverse isoperimetric problem for Gaussian measure. Discrete Comput. Geom., 10(4):411-420, 1993.

[3] T. Bonnesen and W. Fenchel. Theorie der konvexen Körper. Springer-Verlag, Berlin, 1974. Berichtigter Reprint.

[4] P. Bürgisser. Smoothed analysis of condition numbers. In Foundations of Computational Mathematics, Hong Kong 2008, pages 1-41. Cambridge University Press, 2009.

[5] P. Bürgisser, F. Cucker, and M. Lotz. Coverage processes on spheres and condition numbers for linear programming. To appear in Annals of Probability.

[6] P. Bürgisser, F. Cucker, and M. Lotz. Smoothed analysis of complex conic condition numbers. J. Math. Pures et Appl., 86:293-309, 2006.

[7] P. Bürgisser, F. Cucker, and M. Lotz. The probability that a slightly perturbed numerical analysis problem is difficult. Math. Comp., 77(263):1559-1583, 2008.

[8] D. Cheung and F. Cucker. A new condition number for linear programming. Math. Program., 91(1, Ser. A):163-174, 2001.

[9] D. Cheung and F. Cucker. Probabilistic analysis of condition numbers for linear programming. Journal of Optimization Theory and Applications, 114:55-67, 2002 .

[10] D. Cheung, F. Cucker, and R. Hauser. Tail decay and moment estimates of a condition number for random linear conic systems. SIAM J. Optim., 15(4):1237-1261 (electronic), 2005.

[11] F. Cucker, R. Hauser, and M. Lotz. Adversarial smoothed analysis. To appear in $J$. of Complexity. 
[12] F. Cucker and J. Peña. A primal-dual algorithm for solving polyhedral conic systems with a finite-precision machine. SIAM J. Optim., 12(2):522-554 (electronic), 2001/02.

[13] F. Cucker and M. Wschebor. On the expected condition number of linear programming problems. Numer. Math., 94:419-478, 2002.

[14] J. Demmel. The probability that a numerical analysis problem is difficult. Math. Comp., 50:449-480, 1988.

[15] J. Dunagan, D.A. Spielman, and S.-H. Teng. Smoothed analysis of condition numbers and complexity implications for linear programming. Math. Programm. Series A, 2009. To appear.

[16] S. Glasauer. Integralgeometrie konvexer Körper im sphärischen Raum. PhD thesis, Universität Freiburg im Br., 1995.

[17] J.-L. Goffin. The relaxation method for solving systems of linear inequalities. Math. Oper. Res., 5(3):388-414, 1980.

[18] R. Hauser and T. Müller. Conditioning of random conic systems under a general family of input distributions. Found. Comput. Math., 9(3):335-358, 2009 .

[19] R. Howard. The kinematic formula in Riemannian homogeneous spaces. Mem. Amer. Math. Soc., 106(509):vi+69, 1993.

[20] J. Renegar. Some perturbation theory for linear programming. Math. Programming, 65(1, Ser. A):73-91, 1994.

[21] J. Renegar. Incorporating condition measures into the complexity theory of linear programming. SIAM J. Optim., 5(3):506-524, 1995.

[22] J. Renegar. Linear programming, complexity theory and elementary functional analysis. Math. Programming, 70(3, Ser. A):279-351, 1995.

[23] A. Sankar, D.A. Spielman, and S.H. Teng. Smoothed analysis of the condition numbers and growth factors of matrices. SIAM J. Matrix Anal. Appl., 28(2):446-476 (electronic), 2006.

[24] R. Schneider. Smooth approximation of convex bodies. Rend. Circ. Mat. Palermo (2), 33(3):436-440, 1984.

[25] S. Smale. Complexity theory and numerical analysis. In A. Iserles, editor, Acta Numerica, pages 523-551. Cambridge University Press, 1997.

[26] D.A. Spielman and S.-H. Teng. Smoothed analysis of algorithms. In Proceedings of the International Congress of Mathematicians, volume I, pages 597-606, 2002 .

[27] D.A. Spielman and S.-H. Teng. Smoothed analysis of termination of linear programming algorithms. Math. Programm. Series B, 97:375-404, 2003. 
[28] D.A. Spielman and S.-H. Teng. Smoothed analysis: Why the simplex algorithm usually takes polynomial time. Journal of the ACM, 51:385-463, 2004.

[29] M. Spivak. A comprehensive introduction to differential geometry. Vol. III. Publish or Perish Inc., Wilmington, Del., second edition, 1979.

[30] R. Webster. Convexity. Oxford Science Publications. The Clarendon Press Oxford University Press, New York, 1994.

[31] J. G. Wendel. A problem in geometric probability. Math. Scand., 11:109-111, 1962.

[32] H. Weyl. On the Volume of Tubes. Amer. J. Math., 61(2):461-472, 1939. 